Aus dem Department für Nutztierwissenschaften

- Abteilung Verfahrenstechnik in der Veredelungswirtschaft -

der Georg-August-Universität Göttingen

Fakultät für Agrarwissenschaften

\title{
Variationen im Auslaufmanagement von Sportpferden in Einzelhaltung: Beurteilung der Auswirkungen auf die Tiergerechtheit an Hand des Tierverhaltens und der Stressbelastung
}

\author{
Dissertation \\ zur Erlangung des Doktorgrades \\ der Fakultät für Agrarwissenschaften \\ der Georg-August-Universität Göttingen
}

vorgelegt von

Hanna Werhahn

geboren in Uelzen

Göttingen, Juli 2011 
D7

1. Referentin: Prof. Dr. Engel F. Hessel

2. Korreferent: $\quad$ Prof. Dr. Dr. Matthias Gauly

Tag der mündlichen Prüfung: 21. Juli 2011 


\section{Inhaltsverzeichnis}

Inhaltsverzeichnis

Abkürzungsverzeichnis................................................................... III

1. Einleitung und Zielsetzung ..................................................... 1

2. Stand des Wissens .................................................................. 2

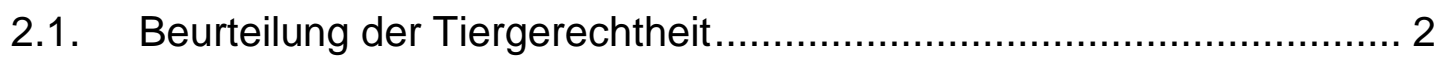

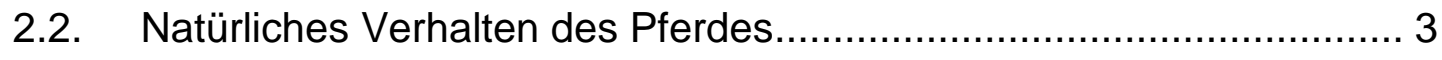

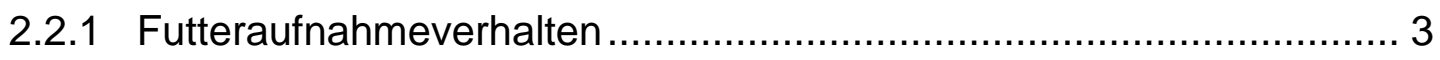

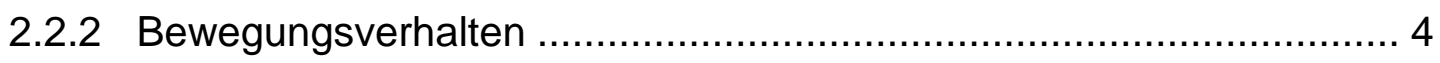

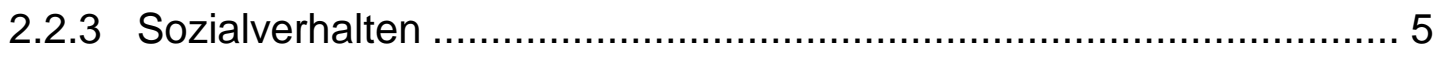

$\underline{2.2 .4 \text { Ruheverhalten } \ldots \ldots \ldots \ldots \ldots \ldots \ldots \ldots \ldots \ldots \ldots \ldots \ldots \ldots \ldots \ldots \ldots \ldots \ldots \ldots \ldots \ldots \ldots \ldots \ldots \ldots \ldots \ldots \ldots \ldots \ldots \ldots \ldots \ldots \ldots}$

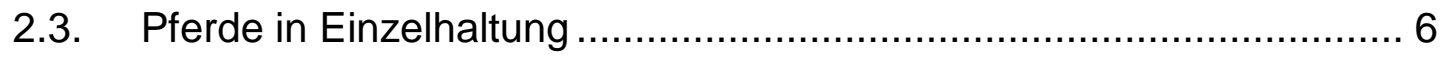

2.3.1 Gründe für die Wahl der Einzelboxenhaltung $\ldots \ldots \ldots \ldots \ldots \ldots \ldots \ldots \ldots \ldots \ldots$

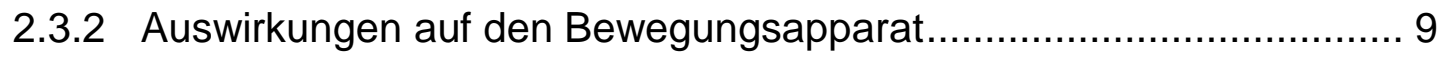

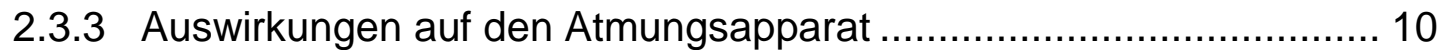

2.3.4 Auswirkungen auf den Verdauungsapparat ........................... 11

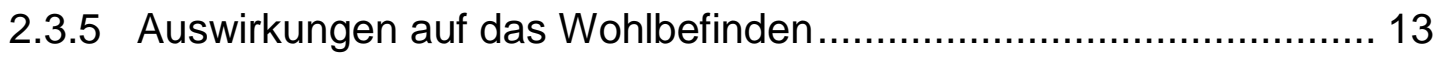

2.4. Methoden zur Erfassung des Tierverhaltens und der

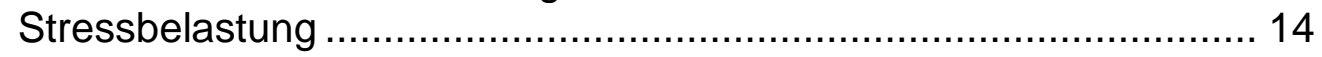

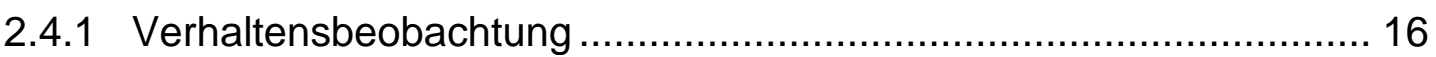

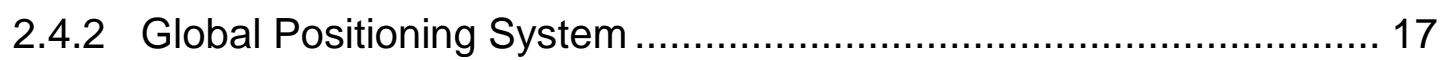

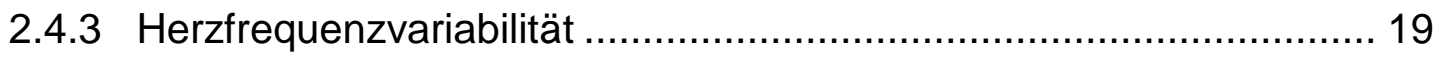

3. Beiträge mit Begutachtung …............................................... 24

3.1. Temporary turnout for free exercise in groups: effects on the behaviour of competition horses housed in single stalls ............... 24

3.2. Competition horses housed in single stalls (1st communication): Behaviour and activity patterns during free exercise according to

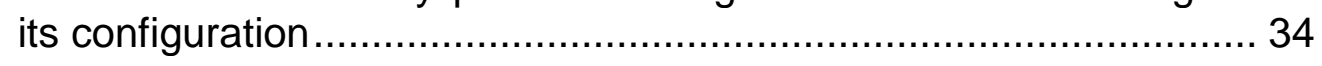

3.3. Competition horses housed in single stalls (2nd communication): Effects of free exercise on the behaviour in the stable and during

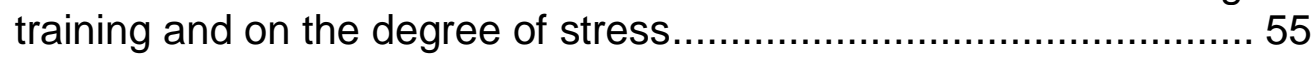

4. Diskussion............................................................................ 82

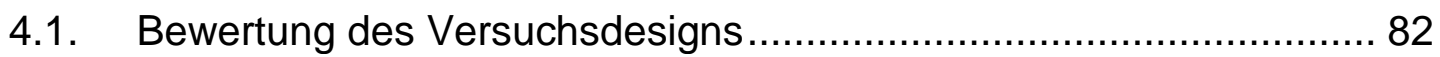

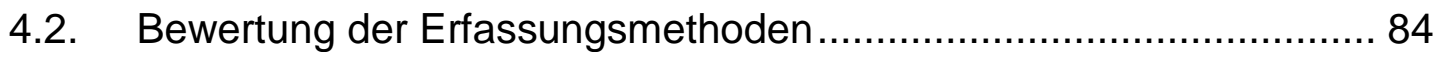

4.3. Erkenntnisse aus den Managementvarianten über die Tiergerechtheit der Einzelhaltung von Pferden ......................... 86 


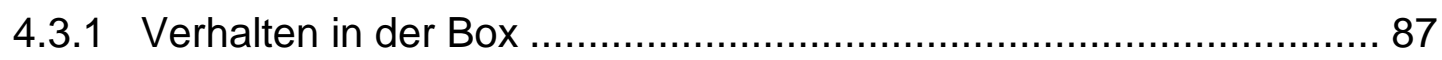

4.3.2 Verhalten und Bewegungsaktivität im Auslauf ................................ 89

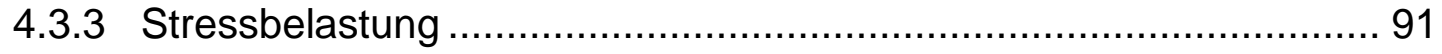

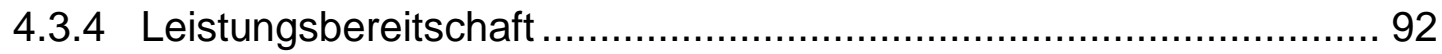

5. Zusammenfassung .................................................................... 94

6. Summary.................................................................................... 97

7. Literaturverzeichnis .................................................................

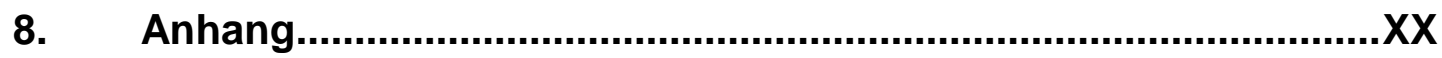

8.1. Bewertungsbögen ...............................................................

8.1.1 Verhalten während des Auslaufs ..............................................XX

8.1.2 Verhalten während des Trainings ............................................XI

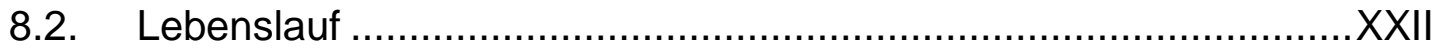

8.3. Veröffentlichungen ......................................................... XXIV

8.3.1 Wissenschaftliche Beiträge mit Begutachtung ……................... XXIV

8.3 .2 Tagungsbeiträge .......................................................... XXXII

8.3.3 Sonstige Veröffentlichungen ............................................. XXXIII 


\section{Abkürzungsverzeichnis}

HRV heart rate variability (Herzfrequenzvariabilität)

NT no turnout (kein Auslauf)

TBT turnout before training (Auslauf vor dem Training)

TAT turnout after training (Auslauf nach dem Training)

ST solitary turnout (Einzelauslauf)

GT group turnout (Gruppenauslauf)

GPS global positioning system

IBI inter-beat-interval (Intervall zwischen zwei Herzschlägen)

SDNN Standardabweichung aller RR-Intervalle

RMSSD Quadratwurzel des quadrierten Mittelwertes der Summe aller Differenzen sukzessiver RR-Intervalle

LF low frequency (niedrige Frequenz)

HF high frequency (hohe Frequenz)

ANS autonomes Nervensystem

RR-Intervall zeitlicher Abstand zwischen zwei Herzschlägen

EKG Elektrokardiogramm

LM Lebendmasse 


\section{Einleitung und Zielsetzung}

Auch nach etwa 6000-jähriger Domestikation ist das Verhalten des Pferdes noch heute geprägt durch seinen ursprünglichen Lebensraum, die Steppen und Halbwüsten Osteuropas und Zentralasiens. Im Laufe der Zeit haben die natürliche Selektion in unterschiedlichen Lebensräumen und die künstliche Selektion durch den Menschen das äußere Erscheinungsbild des Pferdes zwar verändert, die Ansprüche des Pferdes an seine Umwelt und sein Verhalten sind jedoch erhalten geblieben (Meyer und Coenen, 2002).

Während in dem Bereich der Freizeitreiterei ein Trend zu offeneren Haltungssystemen und zur Gruppenhaltung besteht, überwiegt in dem Bereich der Sportreiterei klar die Einzelboxenhaltung (Korries, 2003; Ahlswede et al., 2005). In beiden Systemen entscheidet das Management über das Wohlbefinden der Tiere und somit über die Tiergerechtheit der Haltungsform (BMELV, 2009).

Ziel der vorliegenden Arbeit war es, gängige Managementvarianten in der Einzelhaltung von Sportpferden im Hinblick auf ihre Auswirkungen auf das Verhalten und die Stressbelastung der Pferde systematisch zu untersuchen. Schwerpunkte der Auswertung lagen sowohl auf den Bedürfnissen des Pferdes (insbesondere nach freier Bewegung und Sozialkontakten) als auch dem Interesse des Reiters bzw. Halters nach einer hohen Leistungsbereitschaft und einer geringen Verletzungsgefahr für das Pferd. Mit den gewonnenen Erkenntnissen sollten Hinweise zur Verbesserung der Tiergerechtheit in dem System der Einzelhaltung erarbeitet werden, ohne die Ansprüche des Reiters bzw. Halters zu vernachlässigen. 


\section{Stand des Wissens}

\subsection{Beurteilung der Tiergerechtheit}

Eine offizielle Definition des Begriffes "Tiergerechtheit" gibt es nicht. Auch im deutschen Tierschutzgesetz taucht dieser Begriff nicht explizit auf. Dennoch heißt es darin, dass Leben und Wohlbefinden des Tieres zu schützen sind (TierSchG §1) und Begriffe wie „artgemäß", „verhaltensgerecht" und „den Bedürfnissen entsprechend" sind wiederholt zu finden, ohne dass sie genauer definiert werden. Seit den 1960er Jahren werden drei wesentliche Kriterien zur Beurteilung der Tiergerechtheit herangezogen. Zum einen handelt es sich um die klinische Gesundheit, physiologische Funktionalität und den Schutz vor äußeren Einflüssen. Diese Parameter können objektiv beurteilt und grundsätzlich auch von der Einzelboxenhaltung von Pferden erfüllt werden. Das zweite Kriterium ist die Möglichkeit der Ausübung der natürlichen Verhaltensweisen (Broom, 1991). Auch dieses Kriterium kann objektiv beurteilt werden. Bei der reinen Einzelboxenhaltung von Pferden ohne freie Bewegungsmöglichkeiten mit Artgenossen können jedoch viele der natürlichen Verhaltensweisen nicht oder nur eingeschränkt ausgeübt werden. Drittens soll der emotionale Zustand des Tieres in die Beurteilung einfließen. Als Maßstab wird hier das menschliche Empfinden angesetzt, welches jedoch aus wissenschaftlicher Sicht nur in sehr begrenztem Maße auf Tiere übertragbar ist (Fraser, 2008). Auf die praktische Pferdehaltung hat das menschliche Empfinden allerdings einen großen Einfluss. Leicht werden dem Tier beispielsweise Empfindungen oder ein Willen unterstellt, um Haltungs- oder Umgangsformen zu rechtfertigen. Ohne ausreichende Kenntnisse über das natürliche Verhalten des Pferdes, fallen solche Unterstellungen für das Pferd jedoch häufig negativ aus (Broom, 1991).

In der Wissenschaft wird versucht, die Tiergerechtheit von Haltungssystemen mit Hilfe von Indikatoren möglichst objektiv zu bestimmen. Als Indikatoren können das Verhalten, Gesundheit, Stress und die Leistung der Tiere dienen. Die Beurteilung erfordert ein großes $\mathrm{Maß}$ an Sachkenntnis in den Bereichen Physiologie und Verhalten und sollte nach Möglichkeit unter Berücksichtigung mehrerer Indikatoren im Zusammenhang mit Einflüssen aus der 
Umwelt erfolgen (Broom, 1991; Brade, 2001; Fraser, 2008). Mit dem Ziel die Beurteilung der Tiergerechtheit zu standardisieren, wurden in den letzten Jahren der Nationale Bewertungsrahmen für Tierhaltungssysteme (KTBL, 2006) und das System Welfare Quality (Keeling, 2009) entwickelt. Im Nationalen Bewertungsrahmen wird der Schwerpunkt auf die baulichen Gegebenheiten als Voraussetzung für die Gesundheit und die Ausführbarkeit der natürlichen Verhaltensweisen gelegt. In dem System Welfare Quality dagegen liegt das Hauptaugenmerk auf der tatsächlichen Auftrittshäufigkeit von Läsionen, Krankheiten und Verhaltensstörungen in dem zu beurteilenden Haltungssystem.

\subsection{Natürliches Verhalten des Pferdes}

Das natürliche Verhalten des Pferdes ist geprägt durch seinen natürlichen Lebensraum. In den weitläufigen Steppen und Halbwüsten Osteuropas und Zentralasiens entwickelte sich das Pferd zu einem hoch spezialisierten Herden-, Lauf- und Fluchttier (McDonnell, 2002). Die starken Temperaturschwankungen insbesondere zwischen Tag und Nacht in diesen Gebieten (Lavrenko und Karamysheva, 1993) führten zu einer guten Anpassungsfähigkeit an wechselnde klimatische Bedingungen. Im Folgenden wird auf die Funktionsbereiche des Verhaltens genauer eingegangen, die für die Einzelhaltung von Pferden von besonderer Bedeutung sind.

\subsubsection{Futteraufnahmeverhalten}

Das Futterangebot in Steppen und Halbwüsten ist begrenzt und besteht überwiegend aus rohfaserreichen, energie- und eiweißarmen Gräsern (Lavrenko und Karamysheva, 1993). Um seinen Ernährungsbedarf zu decken, musste das Pferd große Mengen dieser Nahrung aufnehmen, was dazu führte, dass es sich den größten Teil des Tages mit der Futtersuche und -aufnahme beschäftigte. Entsprechend ist der Verdauungsapparat an die kontinuierliche Zufuhr dieser minderwertigen Nahrung angepasst (Meyer und Coenen, 2002). Das Fassungsvermögen des Magens ist im Verhältnis zum Körper und auch zum restlichen Verdauungssystem mit 5 bis 15 Litern sehr klein. Die hauptsächliche Verdauungsarbeit, die mikrobakterielle Verdauung, 
findet im Dickdarm (großes Kolon) statt, der mit 50 bis 60 Litern Fassungsvermögen große Teile des Bauchraumes ausfüllt (Riegel und Hakola, 2002). Noch heute verbringen frei lebende Pferde zwischen 45 und $75 \%$ des Tages mit der Futteraufnahme, wobei die Dauer maßgeblich von der Futterverfügbarkeit abhängt (Kownacki et al., 1978; Duncan, 1980; Boyd und Bandi, 2002; Isenbügel, 2002). Die Futteraufnahme erfolgt sowohl am Tag als auch in der Nacht, wobei die Witterungsbedingungen großen Einfluss auf die Hauptfresszeiten haben (Mayes und Duncan, 1986). Fressintervalle werden von maximal zweistündigen Fresspausen unterbrochen (Krull, 1984). Eine ausgeprägt selektive Fressweise ermöglicht es dem Pferd auch bei insgesamt schlechter Futterqualität die größtmögliche Nährstoffmenge aufzunehmen (Meyer und Coenen, 2002).

\subsubsection{Bewegungsverhalten}

Das Bewegungsverhalten des Pferdes ist sehr stark mit dem Nahrungsaufnahmeverhalten verknüpft. Da das Futterangebot in den natürlichen Lebensräumen begrenzt und das Pferd ständig auf der Suche nach der bestmöglichen Futterqualität ist, findet die Nahrungsaufnahme in Bewegung statt und zwar überwiegend im langsamen Schritt. Die Körperhaltung beim Grasen - Ausfallschritt und gesenkter Kopf-Hals-Bereich - ist also für den Großteil des Tages physiologisch (Houpt, 2005). Je nach Verfügbarkeit von Futter und Wasser legen frei lebende Pferde zwischen sechs und 30 Kilometern pro Tag zurück (Frentzen, 1994; Marten, 1998; Bender, 1999; Fraser und Broom, 2004; Zeitler-Feicht, 2008). Die Gangarten Trab und Galopp nehmen bei erwachsenen Pferden unter natürlichen Lebensbedingungen nur einen sehr geringen Teil, weniger als ein Prozent, des Zeitbudgets ein (Kusonose et al., 1987; Houpt, 2005). Trab wird im Rahmen des Erkundungsverhaltens und des Imponierverhaltens bei der Fortpflanzung gezeigt. Galopp wird nur sehr kurz ausgeführt, zum Beispiel im Rahmen des Flucht- und Spielverhaltens. Bei Jungtieren und Hengsten ist im Tagesverlauf mehr Trab und Galopp zu beobachten als bei erwachsenen Stuten (Schäfer, 1978). 
Natürlicherweise befindet sich das Pferd also den Großteil des Tages in Bewegung. An diesen Umstand hat sich auch der Bewegungsapparat angepasst. Durch die kontinuierliche leichte Bewegung wird er geschmeidig gehalten, um dann im Bedarfsfall (z.B. Flucht) kurzfristig hohe Leistungen erbringen zu können ohne Schaden zu nehmen (Safran et al., 1988).

\subsubsection{Sozialverhalten}

Das Leben in der Gruppe bietet dem einzelnen Pferd Schutz vor Feinden. Unter natürlichen Bedingungen leben Pferde in Familienverbänden. Diese bestehen in der Regel aus einem Hengst und einer oder mehrerer Stuten mit ihren Fohlen (Klingel, 1972; Tyler, 1972; Schäfer, 1978). Innerhalb des Familienverbandes besteht eine feste Hierarchie. Die ranghöchste Stute führt den Verband sowohl zu Futter-, Wasser- und Ruheplätzen als auch in Fluchtsituationen. Der Hengst steht in der Rangfolge meistens, jedoch nicht immer, über der ranghöchsten Stute (Levine, 2005). Ernsthafte Auseinandersetzungen kommen bei frei lebenden Pferden nur selten und wenn überhaupt in der Paarungszeit vor. Konflikte werden in der Regel durch ritualisierte Signale der Körpersprache (Drohen) geklärt (Schäfer, 1978; McDonnell, 2002). Die gegenseitige soziale Hautpflege ist einerseits Teil des Komfortverhaltens (z.B. Entfernen loser Haare, Massage schwer erreichbarer Körperstellen, Vertreiben von Ungeziefer), andererseits hat sie auch eine wichtige gruppenbildende und -bindende Funktion. Innerhalb des Familienverbandes bilden sich häufig feste Freundschaften zwischen Tieren ähnlichen Ranges, die dann auch bei anderen Verhaltensweisen (z.B. Grasen, Ruhen) besonders geringe Individualabstände zeigen (Tyler, 1972; Clutton-Brock et al., 1976).

\subsubsection{Ruheverhalten}

Das Ruheverhalten nimmt bei erwachsenen, frei lebenden Pferden 15 bis 25 $\%$ des Tages ein (Duncan, 1980; Boyd et al., 1988). Dabei können verschiede Intensitätsgrade des Ruhens unterschieden werden. Das Dösen, die häufigste Ruheform (15-20 \% des Tages), findet im Stehen statt (Duncan, 1980; Boyd et al., 1988). Auch Phasen des SWS-Schlafes (slow-wavesleep, flacher Schlaf) können im Stehen erreicht werden, meist findet diese 
Ruheform aber in Bauchlage statt. Für Tiefschlafphasen (REM-Schlaf, rapideye-movement) begibt sich das Pferd meist in die Seitenlage (Hale und Huggins, 1980). Insgesamt werden etwa 5-7 \% des Tages im Liegen verbracht (Duncan, 1980; Kiley-Worthington, 1987; Boyd et al., 1988). Aus dem Dösen im Stehen erwacht das Pferd sehr leicht und ist somit schnell fluchtbereit. Das Ablegen erfolgt nur, wenn ein ausreichendes Sicherheitsgefühl vorhanden ist. Das hat zur Folge, dass selten alle Pferde einer Gruppe gleichzeitig liegen (Zeeb, 1981). Die Ruhephasen sind über den gesamten Tag verteilt, wobei das Liegen hauptsächlich in der Nacht - zwischen Mitternacht und Sonnenaufgang - stattfindet (Schäfer, 1978; Boyd et al., 1988). Als Schlafplätze werden vorzugsweise windige Stellen mit trockenem Untergrund und guter Übersicht über die Umgebung gewählt, um dem Sicherheitsbedürfnis der liegenden Pferde genüge zu tun. Zum Dösen werden auch Bereiche aufgesucht, die je nach Witterung Schutz vor Sonne oder Regen bieten (Schäfer, 1978).

\subsection{Pferde in Einzelhaltung}

Die häufigste Form der Pferdehaltung in Deutschland ist die Einzelboxenhaltung. Untersuchungen ergaben, dass $99 \%$ der Sportpferde in Niedersachsen (Korries, 2003), $90 \%$ der Pensionspferde in Schleswig-Holstein (Petersen et al., 2006), $80 \%$ der Großpferde in Deutschland (Ahlswede et al., 2005) und etwa $68 \%$ aller Pferde in Deutschland (Tigges, 2009) in Einzelhaltung untergebracht sind. Diese Haltungsform hat große Vorteile im Hinblick auf die Überwachung, Versorgung und auch die Verfügbarkeit des Einzeltieres. Andererseits wird das natürliche Verhalten des Pferdes sehr stark eingeschränkt. Eine tiergerechte Gestaltung des Systems ist bei geeigneten Rahmenbedingungen und Managementmaßnahmen zwar möglich, in der Regel aber auch mit erhöhtem Arbeitsaufwand verbunden (Haidn et al., 2002; Borstel et al., 2010). Experten fordern eine Mindestboxengröße von $(2 \times \text { Widerristhöhe })^{2}$, mindestens Sicht-, Hör- und Geruchskontakt zu Artgenossen und den physiologischen Ansprüchen entsprechende Bewegung (BMELV, 2009). 
Unklar ist allerdings, wie viel Bewegung und welche Bewegungsform konkret den physiologischen Ansprüchen entsprechen. Meist wird dieser Anspruch vom natürlichen Verhalten abgeleitet. Allerdings wird gerade die Bewegungsaktivität in Freiheit entscheidend von der Futterverfügbarkeit und auch von Feinden (Flucht) bestimmt. Beides wird in menschlicher Obhut kontrolliert und erfordert in der Regel keine Bewegung (Elia, 2002; Goodwin et al., 2002). Der tatsächliche Bewegungsbedarf im Hinblick auf das physiologische und psychologische Wohlbefinden ist also nicht bekannt. Entsprechend vielfältig ist das Spektrum der Bewegungsangebote in der Praxis. Im Bereich der Einzelhaltung reicht es von reiner Boxenhaltung ohne freie Bewegungsmöglichkeiten bis hin zu 24-stündigem Weide-/Paddockaufenthalt. Zeitlich begrenzte Auslaufaufenthalte wiederum sind sowohl einzeln als auch in Gruppen zu finden, wobei im Hinblick auf Gruppengröße und -zusammensetzung sämtliche denkbare Konstellationen praktiziert werden (Korries, 2003; Ahlswede et al., 2005; Petersen et al., 2006; Tigges, 2009).

\subsubsection{Gründe für die Wahl der Einzelboxenhaltung}

Experten sind sich darin einig, dass die reine Boxenhaltung mit täglichem Training und ohne zusätzliche freie Bewegungsmöglichkeiten nicht tiergerecht ist. Einschränkend wird jedoch darauf hingewiesen, dass eine solche Haltungsform toleriert werden kann, wenn das entsprechende Tier bei freier Bewegung eine Gefahr für sich und andere darstellt (BMELV, 2009). Letztlich obliegt es also dem Halter zu entscheiden, für welche Haltungsform das individuelle Tier geeignet ist.

In der Praxis kommen noch weitere Faktoren hinzu, die die Haltungsform (in diesem Fall die Einzelboxenhaltung) und deren Gestaltung beeinflussen. Die Haltung und der Handel mit Pferden, insbesondere mit Sportpferden, dienen vielen Reitern, Ausbildern, Händlern und auch Landwirten als Lebensgrundlage oder zumindest als Einnahmequelle (Ipsos, 2001). Die entscheidende Voraussetzung für eine zufriedene Kundschaft und somit für das Geschäft sind gesunde Pferde. Die Form der Einzelhaltung ist zwar vergleichsweise teuer und aufwendig (viel Platzbedarf/Pferd im Vergleich zur Gruppenhaltung; Abtrennungen, Futtertröge, Tränken, Füttern, Einstreuen, 
Entmisten in jeder Box), andererseits ist die Überwachung und individuelle Behandlung (insbesondere die Fütterung) der Pferde in diesem System sehr gut möglich. Um freie Bewegung gewähren zu können, muss der Betrieb zunächst über geeignete Flächen verfügen. Sind diese vorhanden, muss er die Arbeitszeit für die Verbringung der Pferde auf die Flächen aufbringen (Marten, 1998).

Jede Art von Bewegung bietet ein gewisses Risiko für Verletzungen. Während des Trainings mag es gering oder zumindest vom Trainierenden beeinflussbar sein. Bei freier Bewegung ist eine Einflussnahme kaum möglich, was die Verletzungsgefahr erhöht. Insbesondere bei unregelmäßiger freier Bewegung ist häufig eine sehr intensive Bewegungsaktivität („Austoben“) zu Beginn des Auslaufs zu beobachten (Kusonose et al., 1987; Hogan et al., 1988; Mal et al., 1991; Houpt et al., 2001; Chaya et al., 2006), welche die Verletzungsgefahr deutlich ansteigen lässt, da der Bewegungsapparat durch das Stehen in der Box nicht elastisch ist (Safran et al., 1988). Da Pferde sowohl einen hohen ideellen, aber durchaus auch einen hohen monetären Wert darstellen können (Icken et al., 2007), wird versucht, das Verletzungsrisiko möglichst gering zu halten. Dieses führt häufig zu einer Einschränkung oder zu einem nicht Gewähren von freier Bewegung.

Auch die Zeit, die der Reiter für die Beschäftigung mit dem Pferd ansetzt, wird von der Haltungsform beeinflusst. Steht das Pferd in der Einzelbox, ist es leicht verfügbar und - bei entsprechender Pflege der Einstreu - verhältnismäßig sauber. Die benötigte Zeit zur Vor- und Nachbereitung des Trainings ist also gering (Schäfer, 1991).

Die Haltung von Sportpferden in Einzelboxen ohne freie Bewegungsmöglichkeiten wird in der Praxis häufig auch mit persönlichen Ansichten oder Erfahrungen an Stelle von sachlichen Argumenten gerechtfertigt. Obwohl bereits wissenschaftlich widerlegt (Jørgensen und Bøe, 2007), besteht beispielsweise die These, dass Pferde durch das Training ausreichend Bewegung bekommen und somit zusätzlicher Auslauf nicht notwendig ist. Auch wird teilweise befürchtet, dass die Leistungsbereitschaft der Pferde durch freie Bewegungsmöglichkeiten sinken würde, da dann im Training keine Motivation zur Bewegung mehr bestehen würde (Houpt und 
McDonnell, 1993). Da die Beurteilung der Leistungsbereitschaft sehr subjektiv ist, ist eine wissenschaftliche Klärung dieses Zusammenhanges schwierig. Bereits nachgewiesen ist jedoch, dass die freie Bewegung positive Auswirkungen auf die Ausdauerleistungsfähigkeit von Pferden hat (Arnemann, 2003).

\subsubsection{Auswirkungen auf den Bewegungsapparat}

Für das Pferd überwiegen in dem System der Einzelhaltung ohne freie Bewegung die Nachteile, die bei genauem Hinsehen auch für den Halter langfristig zum Problem werden können. Wird dem Pferd zusätzlich zum Training keine freie Bewegung gewährt, verbringt es etwa $95 \%$ des Tages mit Stehen oder Liegen und nur etwa $5 \%$ mit Bewegung (Kiley-Worthington, 1987). Verglichen mit dem Verhalten unter natürlichen Bedingungen (Kapitel 2.1) handelt es sich hier um eine starke Abweichung vom natürlichen Zeitbudget. Studien haben ergeben, dass im Training zwischen 3 und $10 \mathrm{~km}$ zurückgelegt werden (Rodewald, 1989; Widmann, 1990). Ähnliche Strecken wurden durchaus auch bei frei lebenden Pferden gemessen (Fraser und Broom, 2004), jedoch wird diese Strecke im Training in einem vergleichsweise sehr kurzen Zeitrahmen zurückgelegt und vorwiegend in den Gangarten Trab und Galopp (Rodewald, 1989). Die Kombination von Einzelboxenhaltung mit Training kann also zu einer zurückgelegten Strecke führen, die der in Freiheit ähnlich ist, sie entspricht aber nicht dem physiologischen Bewegungsverhalten des Pferdes. Aus physiologischer Sicht führt die lange Stehzeit zu einer Minderung der Elastizität des Bewegungsapparates (Safran et al., 1988). Bei kontinuierlicher langsamer Bewegung wird über den Hufmechanismus ständig die Durchblutung der Extremitäten angeregt (Douglas et al., 1996), was den Bewegungsapparat elastisch hält. Er ist somit bei Bedarf belastbar (Shellock und Prentice, 1985). Die erhöhte Stehzeit in der Einzelbox führt zu einer verminderten Durchblutung der Extremitäten, was ihre Elastizität und somit die Belastbarkeit deutlich einschränkt. Wird der Bewegungsapparat vor einer Belastung (Training, Auslauf) nicht sorgfältig aufgewärmt, entsteht eine sehr hohe Verletzungsgefahr (Shellock und Prentice, 1985; Safran et al., 1988), was 
sowohl für das Pferd als auch für den Halter nachteilig ist. Schäden am Bewegungsapparat sind mit 40 bis $61 \%$ (je nach Untersuchung) die häufigste Ursache für die Unbrauchbarkeit für Pferde im Reitsport und haben somit eine sehr große Bedeutung für die Praxis (Butler und Armbruster, 1984; Clausen et al., 1990; Lindner und Offeney, 1992; Hommerich, 1995; Wallin et al., 2000).

\subsubsection{Auswirkungen auf den Atmungsapparat}

Ein weiteres besonders leistungsfähiges aber auch ebenso empfindliches System im Körper des Pferdes ist der Respirationstrakt. Im Vergleich zu anderen Säugetieren ist die Lunge des Pferdes im Verhältnis zu seinem Körper besonders groß (Volumen bei $600 \mathrm{~kg}$ Körpermasse etwa 60 Liter; Gehr et al., 1981). Entsprechend groß sind auch die Sauerstoffaufnahmekapazität und der Verbrauch von Sauerstoff im Stoffwechsel (Gehr und Erni, 1980). Bei ausreichend vorhandener frischer Luft ist der Respirationstrakt in der Lage, sich selbst zu reinigen. Dieses geschieht insbesondere bei der erhöhten Atemfrequenz in Bewegung (Lekeux und Art, 1994). Wenig Bewegung führt entsprechend auch zu einer Belastung des Atmungsapparates. Zusätzlich zu unzureichender Bewegung ist auch die Luftqualität in vielen Ställen vermindert (Jussen et al., 1984; Bracher, 1987; Vandenput et al., 1998; Gerber et al., 2003). Experten sind sich einig, dass Lufttemperatur und Luftqualität (relative Luftfeuchtigkeit, Staubgehalt und Gehalt an Schadgasen wie Ammoniak, Kohlenstoffdioxid und Schwefelwasserstoff) den Außenbedingungen gemäßigt folgen sollen (BMELV, 2009). Dennoch ist die Luftqualität in vielen Ställen mangelhaft (Holcombe et al., 2001; Korries, 2003). Schlechte Belüftung kann einerseits durch bauliche Gegebenheiten begründet sein, insbesondere bei Altgebäuden. Andererseits wird eine gute Belüftung teilweise bewusst verhindert, beispielsweise um das Einfrieren von Wasserleitungen im Winter zu vermeiden. Zum Teil werden auch menschliche Maßstäbe an ein „angenehmes Klima“ angesetzt, was sich in erster Linie auf die Temperatur bezieht (Marten, 2000). Dieses führt meist $z u$ einer zu starken Abpufferung der Außenbedingungen (insbesondere im Hinblick auf die Temperatur) für das Pferd, das auf Grund seiner Herkunft 
sehr gut mit starken Temperaturschwankungen umgehen kann (vgl. Kapitel 2.1) und eine sehr weite thermoneutrale Zone besitzt (Spanne von etwa $2^{\circ} \mathrm{C}$, je nach Gewöhnung zwischen $-15^{\circ}$ bis $+10^{\circ} \mathrm{C}$ (Mc Bride et al., 1985), zwischen $-10^{\circ}$ und $+15^{\circ} \mathrm{C}$ (Martin-Rosset und Vermorel , 1991) bzw. zwischen $+5^{\circ}$ und $+25^{\circ} \mathrm{C}$ (Morgan et al., 1997; Morgan, 1998)) . Die schädigende Wirkung von Stäuben und Schadgasen in der Stallluft wird häufig unterschätzt, was sich an der Häufigkeit von Atemwegserkrankungen zeigt. Holcombe et al. (2001) stellten eine signifikant größere Belastung der Atemwege bei Stallhaltung im Vergleich zur Weidehaltung fest und auch Korries (2003) ermittelte einen direkten Zusammenhang zwischen dem Stallklima und dem Auftreten respiratorischer Erkrankungen. Bei 9 bis $17 \%$ der Pferde haben solche Erkrankungen zur Schlachtung geführt (Clausen et al., 1990; Lindner und Offeney, 1992; Hommerich, 1995; Wallin et al., 2000). Schlichting (2001) vermutet, dass bei über $30 \%$ der untersuchten Pferde eine Unbrauchbarkeit für den Reitsport durch Schädigungen der Atemwegsorgane begründet ist und Pick (1986) prognostizierte sogar bei etwa $80 \%$ der im Stall gehaltenen Pferde symptomatische Atemwegserkrankungen.

\subsubsection{Auswirkungen auf den Verdauungsapparat}

Wie in Kapitel 2.1 beschrieben, ist der Verdauungstrakt des Pferdes für eine kontinuierliche Aufnahme von rohfaserreicher und nährstoffarmer Nahrung ausgelegt. Die Praxis der Fütterung in Einzelhaltung, insbesondere von Sportpferden, weist ein sehr weites Spektrum auf, welches von der ad libitum Verfügbarkeit von Raufutter bis hin zur fast ausschließlichen Gabe von Kraftfutter reicht. Empfohlen werden täglich mindestens $1 \mathrm{~kg}$ Raufutter/100kg LM und pro Mahlzeit maximal 0,5 kg Kraftfutter/100 kg LM (Meyer und Coenen, 2002). Bei Sportpferden ist häufig eine Überversorgung mit Kraftfutter in Kombination mit einer Unterversorgung mit Raufutter zu beobachten, die zu Problemen in der Verdauungsphysiologie führen kann (siehe unten). Die Ursachen für solche Fütterungsfehler sind vielfältig. Unwissenheit oder eine Überschätzung der vom Pferd geleisteten Arbeit können Gründe sein. Zusätzlich ist Raufutter deutlich voluminöser als 
Kraftfutter. Das führt dazu, dass zwar der Anschaffungspreis je Kilogramm im Vergleich zu Kraftfutter geringer ist, dafür aber Werbung (Transporte), Lagerung (Raum muss vorhanden sein) und auch Vorlage (vom Lagerplatz zum Pferd, Reinigung nach der Vorlage) deutlich aufwendiger und somit teurer sind.

Aus physiologischer Sicht führt Kraftfutter im Vergleich zu Raufutter zu einer deutlich geringeren Kauaktivität je Gewichtseinheit, was einerseits zu verminderter Fresszeit (zur ethologischen Bedeutung des Fressens siehe Kapitel 2.3.5) und andererseits zu geringerer Speichelproduktion führt (Ellis, 2003). Eine ausreichende Zerkleinerung des Futters durch das Kauen ist notwendig, um die Angriffsfläche für Enzyme und Mikroben zu erhöhen und so die enthaltenen Nährstoffe aufzuschließen. Obwohl im Speichel des Pferdes keine Enzyme enthalten sind, ist eine ausreichende Einspeichelung notwendig, um das Futter einzuweichen, schluckfähig zu machen und eine gute Durchdringung mit den Verdauungssäften des Magens zu ermöglichen (Meyer und Coenen, 2002).

Die Regulation der Futteraufnahme im Hinblick auf die Beendigung von Mahlzeiten erfolgt bei frei lebenden Pferden (faserreiche Futtermittel) vermutlich in erster Linie durch Ermüdung der Kaumuskulatur (Ralston et al., 1983; Meyer und Coenen, 2002). Dieser Regulationsmechanismus greift nicht, wenn strukturarme Futtermittel (Kraftfutter oder zerkleinerte Raufuttermittel) angeboten werden. Da der Magen des Pferdes keine Dehnungsrezeptoren besitzt, die bei übermäßiger Füllung die Nahrungsaufnahme blockieren, führen große Kraftfuttermengen (und auch andere konzentrierte strukturarme Futtermittel wie z.B. junges Gras) leicht zu Magenüberladungen. In diesem Fall wird der Futterbrei im Magen nicht ausreichend durchsäuert. Das wiederum führt zu einer starken Vermehrung von Mikroorganismen im Magen (die auch unter physiologischen Bedingungen im vorderen drüsenlosen Bereich den Magens (Blindsack, pars oesophagica) aktiv sind) und entsprechenden Fehlgärungen (Gasbildung, überhöhte Milchsäurebildung bis hin zur Schleimhautreizung), die sich in Koliken äußern können. Ähnliche Wirkungen haben auch verdorbene und verkleisternde (stärkereiche aber strukturarme Futtermittel wie z.B. Weizen 
oder Roggen) Futtermittel. Bei psychischen und physischen Belastungen (z.B. starke Belastung direkt nach der Fütterung, Aufregung) kann die autonome Regulation der Verdauungstätigkeit (Motorik und Sensorik: durch Parasympathikus gefördert, durch Sympathikus gehemmt) soweit gehemmt werden, dass es ebenfalls zu Fehlgärungen kommt. Fehlgärungen können wiederum Magengeschwüre auslösen (Meyer und Coenen, 2002). Laut Ahlswede et al. (2005) sind z.B. bei 60-93\% der Vollblüter in der Hochleistungsphase und über $60 \%$ der Turnier- und Distanzpferde Läsionen im Bereich des Magens zu finden.

Übermäßige Kraftfutteraufnahme hat auch Folgen für den restlichen Verdauungstrakt. Entsprechend fließen verhältnismäßig viele leicht verdauliche Substanzen in das Caecum (Blinddarm), wodurch auch in diesem Bereich Fehlgärungen entstehen, die zu Koliken führen können. Der mikrobielle Abbau führt zusätzlich zur Bildung großer Mengen flüchtiger Fettsäuren und Milchsäure. In schweren Fällen kann es zu einer Änderung der Mikrobenzusammensetzung kommen. Durch die verstärkte Milchsäurebildung fällt der $\mathrm{pH}-$ Wert $\mathrm{ab}$, was zu Reizungen der Darmschleimhaut, Motilitätsstörungen und sogar zum Absterben der Mikroorganismen führen kann. Wenn Mikroorganismen absterben, können Endotoxine freigesetzt werden, die bei Absorption zur Entwicklung einer Hufrehe führen können (Meyer und Coenen, 2002).

\subsubsection{Auswirkungen auf das Wohlbefinden}

Die in Einzelhaltung verbreitete rationierte Fütterung mit geringen Raufutterund hohen Kraftfuttergaben verkürzt die Fresszeit und hat somit auch Auswirkungen auf das Wohlbefinden des Pferdes. Während es in Freiheit den größten Teil des Tages mit der Futtersuche und Futteraufnahme beschäftigt ist, nimmt dieser Teil des Zeitbudgets bei rationierter Fütterung in Einzelhaltung einen deutlich geringeren Anteil an. Je nach Fütterungsprinzip sinkt der Anteil des Fressens von $60 \%$ in Freiheit (Duncan, 1980) auf $47 \%$ in Einzelhaltung mit ad libitum Raufutterzugang und auf nur noch $15 \%$ bei rationierter Raufuttergabe (Kiley-Worthington, 1987). Mit der verminderten Fresszeit wird dem Pferd eine wesentliche Beschäftigungsmöglichkeit 
genommen (Elia et al., 2010) und die frei gewordene Zeit wird hauptsächlich mit Stehen ausgefüllt (Kiley-Worthington, 1990). Je nach Gestaltung der Einzelboxen sind auch der Kontakt zu Artgenossen und die Teilnahme am Umweltgeschehen mehr oder weniger stark eingeschränkt, was insgesamt zu einer Reizarmut des Umfeldes führt. Die daraus resultierende Langeweile und Frustration, da das natürliche Verhaltensrepertoire nicht ausgeführt werden kann, können leicht zu Verhaltensanomalien (z.B. besondere Aggressivität oder Passivität) bis hin zu Verhaltensstörungen führen (McGreevy et al., 1995). Verhaltensstörungen sind meist den Funktionskreisen Bewegungsverhalten (z.B. Weben, Boxenlaufen) oder Futteraufnahmeverhalten (z.B. Koppen, Barrenwetzen) zuzuordnen. Hat ein Pferd eine Verhaltensstörung entwickelt, bleibt diese in der Regel auch bei einer Verbesserung der Haltungsumwelt bestehen. Das Risiko zur Entwicklung von Abweichungen vom Normalverhalten kann durch gutes Management (wie z.B. Auslauf, Sozialkontakte, viel Raufuttergabe) auch bei suboptimalen Stallbedingungen deutlich gesenkt werden (Houpt, 2005). Weiterhin sind Temperament und Sensibilität des individuellen Pferdes im Hinblick auf äußere Einflüsse entscheidend für die tatsächliche Ausprägung von Verhaltensstörungen (Dallaire, 1993; Henderson, 2007). Eine genetische Disposition für derartige Entwicklungen wird in der Praxis immer wieder diskutiert, aber weder ein wissenschaftlicher Nachweis noch eine Entkräftung dieser These konnte bisher erbracht werden (Vecchiotti und Galanti, 1987; Dallaire, 1993; Houpt und Kusunose, 2000; Henderson, 2007).

\subsection{Methoden zur Erfassung des Tierverhaltens und der Stressbelastung}

Das Verhalten und die Stressbelastung von Tieren gelten als bedeutende Indikatoren für die Beurteilung des Wohlbefindens von Tieren und somit für die Einschätzung der Tiergerechtheit von Haltungssystemen (Broom, 1991; Fraser, 2008). Um diese Indikatoren zu erfassen, sind verschiedene Methoden etabliert, die jeweils Vor- und Nachteile aufweisen. 
In dem Bereich der Verhaltensbeobachtung werden im Wesentlichen drei Erfassungsschemata unterschieden. Bei der kontinuierlichen Beobachtung werden alle Ereignisse - die von Interesse sind - ohne Unterbrechung mit Auftrittshäufigkeit und Dauer dokumentiert (siehe auch Kapitel 2.4.1). Beim Scan-Sampling-Verfahren (auch Point-Sampling-Verfahren) dagegen wird in festgelegten Zeitintervallen das aktuell ausgeführte Verhalten erfasst. Im Anschluss an die Aufzeichnung wird die Dauer der einzelnen Verhaltensweisen an Hand der Auftrittshäufigkeiten geschätzt (siehe auch Kapitel 2.4.1). Bei dem Verfahren des Instantaneous Sampling (auch 1/0-Verfahren) werden ebenfalls Zeitintervalle festgelegt, wobei dokumentiert wird ob das zu erfassende Verhalten in dem vergangenen Zeitintervall auftrat (,ja“/,1“) oder nicht (,nein“/,0“). Alle drei Verfahren können sowohl direkt (live) als auch indirekt (z.B. Videoaufzeichnung) durchgeführt werden (Gattermann, 2006). Zur Feststellung der Bewegungsaktivität kann einerseits die Verhaltensbeobachtung herangezogen werden (z.B. Unterteilung der beobachteten Fläche mit Hilfe eines Rasters und Erfassung der vom Probanden überquerten Einheiten), andererseits können auch technische Geräte genutzt werden (Gattermann, 2006). Für die Identifikation von Aktivitätsspitzen sind beispielsweise Pedometer geeignet, die an den Extremitäten der Probanden befestigt werden und Erschütterungen erfassen (Brehme et al., 2008). Nach einem ähnlichen Prinzip funktioniert das System Actiwatch $^{\circledR}$ (Activity Monitoring System), das auf einer allseitig empfindlichen (omnidirektionalen) Beschleunigungssensor basiert, der ebenfalls an den Extremitäten befestigt wird (Müller und Schrader, 2003). Um zurückgelegte Strecken und die Ausnutzung von Flächen zu bestimmen, hat sich das Global Positioning System (GPS) bewährt (Tottewitz, 2005; siehe auch Kapitel 2.4.2). Auch drahtlose Sensornetzwerke sollen geeignet sein. Diese bestehen aus Sensorenknoten (,smart dust“ bestehend aus RSS-Sensor (RSS = recieved signal strength), Prozessor, Kommunikationseinheit und Batterie), die an den Probanden befestigt werden, und einer Basisstation. In definierten Zeitabständen treten die Sensorknoten mit der Basisstation in Kontakt, die an Hand der empfangenen Signalstärke die Entfernung des Sensors von der 
Station ermittelt. Mit Hilfe des Abgleichs zwischen aktueller und vorheriger Position kann die Geschwindigkeit berechnet werden (Wark et al., 2007).

Stressbelastungen von Tieren können von erfahrenen Betrachtern durch Abweichungen vom Normalverhalten, also ebenfalls durch Verhaltenbeobachtung, erkannt werden (Broom, 1991). Als technische Möglichkeiten der Evaluierung haben sich die Ermittlung der Herzfrequenzvariabilität (Hottenrott, 2002; siehe auch Kapitel 2.4.3), des Cortisolspiegels im Blut und die Quanitfizierung von Cortisolmetaboliten im Kot (Irvine und Alexander, 1994; Möstl und Palme, 2002) etabliert. Die Eignung des Cortisolspiegels im Speichel für die Feststellung der Stressbelastung von Pferden ist in der Literatur umstritten (Lebelt et al., 1996; Licht, 2000; Elsässer et al., 2001).

\subsubsection{Verhaltensbeobachtung}

\section{Kontinuierliche Beobachtung per Videoaufzeichnung}

Die Auswertung von Verhaltensbeobachtungen mit Hilfe von Videoaufzeichnungen hat den entscheidenden Vorteil, dass die Probanden durch den Beobachter nicht beeinflusst werden können. Weiterhin kann die Geschwindigkeit des Abspielens variiert werden, so dass das Verhalten sehr genau analysiert werden kann. Bei Bedarf kann die Aufzeichnung vor- oder zurückgespult werden, so dass einerseits Erfassungen überprüft und gegebenenfalls korrigiert und andererseits Passagen übersprungen werden können, was eine erhebliche Zeitersparnis bei der Auswertung ermöglicht (Naguib, 2006). Bei der kontinuierlichen Auswertung werden in der Regel sowohl Auftrittshäufigkeit als auch Dauer der Verhaltensweisen erfasst, worüber im Nachhinein auch die mittlere Dauer der Verhaltensweisen bestimmt werden kann (Gattermann, 2006). Nachteilig ist, dass Umwelteinflüsse nur insoweit berücksichtigt werden können, wie sie aus der Aufzeichnung hervorgehen (Naguib, 2006). Software Programme wie "The Observer" (Noldus Information Technology, Wageningen, Niederlande) oder "Interact“ (Mangold International $\mathrm{GmbH}$, Arnstorf) erleichtern die Erfassung und Auswertung der Verhaltensweisen. 


\section{Direkte Beobachtung mit dem Scan-Sampling-Verfahren}

Die direkte Beobachtung des Verhaltens ermöglicht es, Ereignisse in der Umwelt in die Auswertung des Verhaltens einfließen zu lassen. Da das Beobachtungsprotokoll synchron zum Verhalten erstellt wird (digital oder per Hand), ist es möglich, das Verhalten direkt im Anschluss an die Beobachtung zu analysieren. Es muss jedoch berücksichtigt werden, dass der Beobachter, sofern er während der Beobachtung für die Probanden sichtbar ist, das Verhalten unter Umständen beeinflussen kann. Das Scan-SamplingVerfahren ist auf Grund der Erfassungsweise (Momenterfassung, siehe auch Kapitel 2.4) nicht dafür geeignet, kurzfristige Verhaltensweisen, wie beispielsweise Lautäußerungen oder die Frequenz einzelner Bewegungen, zu dokumentieren. Die Erfassung längerfristiger Verhaltensweisen, wie z.B. Fressen oder Liegen ist mit diesem Verfahren jedoch gut möglich, insbesondere da es ohne technische Hilfe einfach durchzuführen ist und eine deutlich geringere Datenmenge generiert als die kontinuierliche Beobachtung. Das Beobachtungsintervall ist frei wählbar und sollte auf die Verhaltensweisen abgestimmt werden, die von Interesse sind. Je kleiner das Beobachtungsintervall gewählt wird, desto genauer kann die Dauer der Verhaltensweisen geschätzt werden (Gattermann, 2006).

\subsubsection{Global Positioning System}

Das Global Positioning System (GPS) ist ein globales Satellitennavigationssystem zur Bestimmung von Positionen. Entwickelt wurde es in den 1970er Jahren für militärische Zwecke in den USA und ist seit 1993 für die weltweite kostenlose Nutzung freigegeben. Das GPS setzt sich aus drei Elementen zusammen. Das Weltraumsegment besteht aus mittlerweile 31 aktiven Satelliten, die auf sechs verschiedenen Umlaufbahnen die Erde in 20180 km Höhe umkreisen. Ein Satellit benötigt für eine Erdumkreisung 12 Stunden und hat einen so genannten „Footprint“ (Ausleuchtzone/ Empfangsbereich des Satelliten) von etwa einem Drittel der Erdoberfläche. Die Satelliten werden vom US-Verteidigungsministerium betrieben und strahlen das für die terrestrische Ortsbestimmung zeitabhängige Signal ab. Zweiter Bestandteil des GPS ist das Kontroll-segment. Dieses setzt sich aus 
elf Bodenstationen zusammen, die so auf der Erde verteilt sind, dass zu jedem Satellit täglich von mindestens vier Stationen aus Sichtkontakt besteht. Von dort aus werden die Satelliten-umlaufbahnen überwacht und die Atomuhren in den Satelliten synchronisiert. Der dritte Bestandteil des GPS ist das Empfangsteil des Nutzers (Anwendersegment), wobei beliebig viele Nutzer die Satellitensignale empfangen können (Mehl, 1996; Zogg, 2009).

Die von den Satelliten ausgesendeten Signale breiten sich mit Lichtgeschwindigkeit aus und benötigen ca. 67 Millisekunden bis zum Empfangsteil. Das Anwendersegment errechnet aus der Zeit, die das Signal vom Aussenden durch den Satelliten bis zum Empfang benötigt, wie weit es sich vom Satelliten entfernt befindet. Vorraussetzung für eine genaue Positionsbestimmung sind absolut synchrone Uhren von Sende- und Empfangssegment. Besteht Kontakt zu mindestens drei Satelliten kann das Empfangsgerät den aktuellen Standort in Längen- und Breitengraden mit Hilfe der Triangulation (Vermessungsnetz auf Basis von Dreiecken) berechnen. Bei Kontakt zu mindestens vier Satelliten kann auch die Höhe des Empfängerstandortes errechnet werden. Durch die ständige Neuberechnung der Position können auch Geschwindigkeit und Bewegungsrichtung berechnet werden. Die Genauigkeit der Positionsbestimmung liegt unter freiem Himmel je nach Gerät zwischen 0,5 und $20 \mathrm{~m}$. Sowohl Niederschlag als auch Bewölkung stören das Signal der Satelliten im Freien kaum. Starke Bewaldung oder Nähe zu hohen Gebäuden können die Genauigkeit der Messung einschränken. In Gebäuden ist je nach Bauart nur schlechter oder kein Signalempfang möglich (Mehl, 1996; Zogg, 2009).

Das GPS ist inzwischen fester Bestandteil in der Land-, Luft- und Seefahrt. Auch der Einsatz als Trainingshilfe im Sport sowohl im Humanbereich als auch im Reitsport (insbesondere Renn-, Fahr- und Vielseitigkeitssport) hat sich etabliert (Liebetrau, 2004; Hebenbrock, 2005; Kingston et al., 2006; Tetzner, 2008). In der Forschung kommt GPS beispielsweise zum Einsatz, wenn die Bewegungsaktivität von Tieren bzw. die Ausnutzung von Flächen bestimmt werden sollen (Hünerbein et al., 2000; Steiner et al., 2000; Turner et al., 2000; Ganskopp, 2001; Kumpula et al., 2001; Bahr, 2007; Lugauer, 2010; Tottewitz et al., 2010). Die Messgenauigkeit wird im Hinblick auf die 
Fortbewegung des Pferdes in Schritt, Trab und Galopp als hoch eingestuft, so dass das System für die Erfassung der Bewegungsaktivität als geeignet betrachtet werden kann (Liebetrau, 2004; Hebenbrock, 2005).

\subsubsection{Herzfrequenzvariabilität}

Seit den 1990er Jahren hat sich die Analyse der Herzfrequenzvariabilität (heart rate variability $=\mathrm{HRV}$ ) und ihre Nutzung als Indikator für die Stressbelastung von Organismen - ausgelöst durch Krankheiten, physische oder psychische Belastungen - etabliert (Task Force, 1996; Hottenrott, 2002; von Borell et al., 2007). Zunächst entwickelt im Bereich der Humanmedizin, wird die Technik inzwischen auch zur Trainingskontrolle im Leistungssport (Hottenrott, 2002) und bei der Beurteilung der Stressbelastung von Tieren eingesetzt (Pferde: Visser et al. (2002); Rietmann et al. (2004), Hoffmann (2008), Nagy et al. (2009). Wiederkäuer: Mohr et al. (2002), Désiré et al. (2004), Hagen et al. (2005). Schweine: de Jong et al. (2000), Geverink et al. (2002), Kuwahara et al. (2004)).

\section{Physiologische Grundlagen}

Die Analyse der HRV basiert auf der Schwankung der zeitlichen Abstände zwischen aufeinander folgenden Herzperioden über einen bestimmten Messzeitraum. Eine Herzperiode beschreibt die typischen Potentialschwankungen des Herzens während der Kontraktion, die im Elektrokardiogramm (EKG) dargestellt werden. Die einzelnen Potenziale werden mit den Buchstaben $P, Q, R, S$ und $T$ bezeichnet (Abb. 1). Die Herzperiodendauer (Abstand zwischen den Herzschlägen) wird an Hand der Abstände zwischen den R-Zacken (RR-Intervall) bestimmt (Hottenrott, 2002).

Die Kontraktion des Herzens wird durch die diastolische Depolarisation (Auslösung des Aktionspotentials zur Kontraktion der Herzmuskelzellen) des Sinusknotens hervorgerufen. Die Häufigkeit der diastolischen Depolarisation und damit die Herzfrequenz (Häufigkeit der Herzkontraktion pro Minute) unterliegen dabei einer Vielzahl zentralnervaler und peripher-reflektorischer Mechanismen und humoraler Einflüsse. 


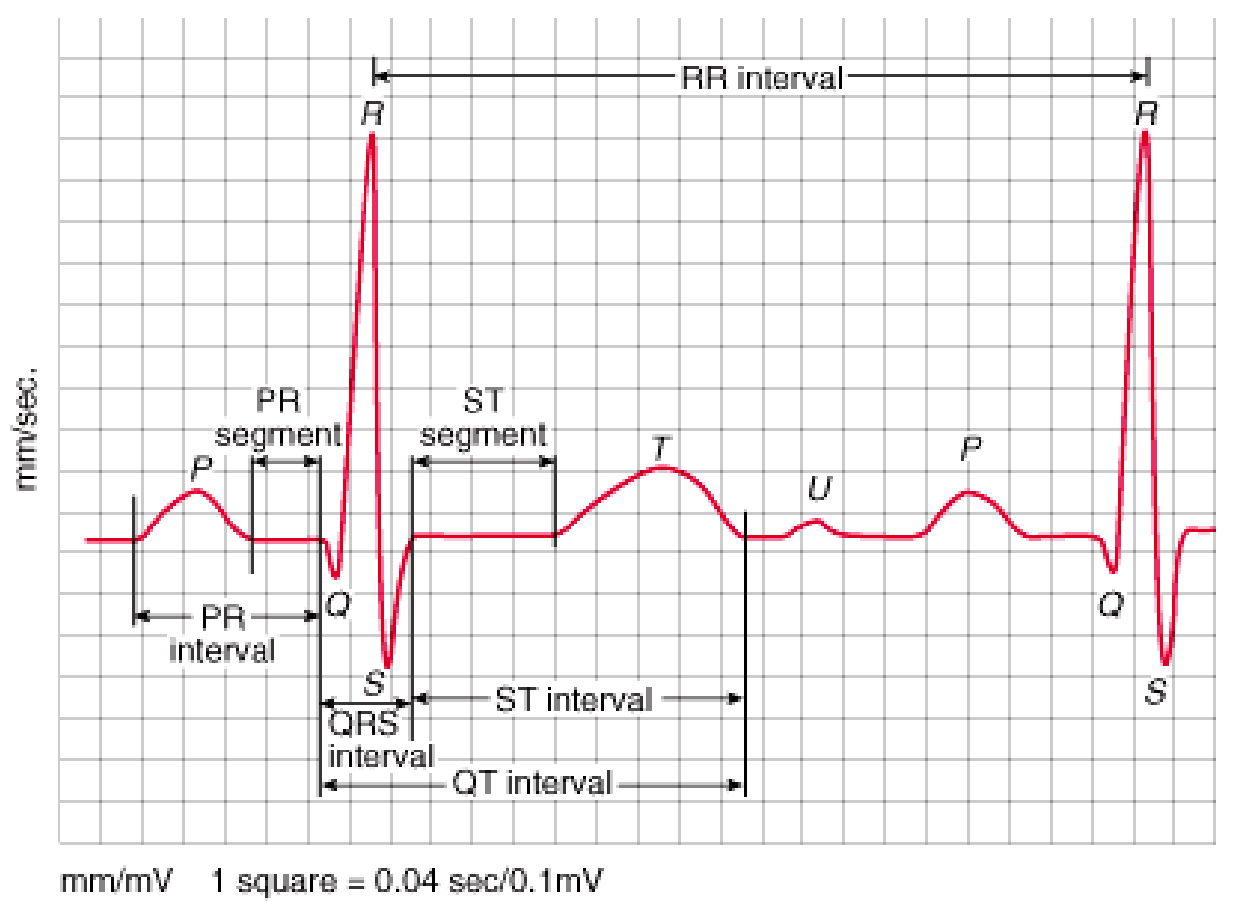

Abb. 1: Schematische Darstellung des Elektrokardiogramms (EKG) mit Bezeichnung der Potenziale (Quelle: http://beslakmal.blogspot.com) .

Von großer Bedeutung ist dabei das Zusammenspiel des sympathischen und parasympathischen Nervensystems, wobei immer beide Systeme tonisch aktiv sind. Der hochfrequente (high frequency $=$ HF) Einfluss des Parasympathikus dominiert in Körperruhe (entspannungsbezogene Wirkung) und führt zu einer Verlangsamung der diastolischen Depolarisation, also zu einer Verringerung der Herzfrequenz. Der niederfrequente (low frequency $=L F$ ) Einfluss des Sympathikus dominiert bei körperlicher Aktivität (z.B. sportlicher Leistung) und führt zu einer Beschleunigung der diastolischen Depolarisation und somit zu einer Beschleunigung der Herzfrequenz. Je nach körperinneren und -äußeren Anforderungen variiert die Stärke der sympathischen und parasympatischen Stimulation ständig, was zu der natürlichen Variation der Herzperiodendauer führt. Die HRV ist also eine Kenngröße, die die Fähigkeit des Herzens beschreibt, sich an endogene und exogene Belastungsfaktoren anzupassen (Hottenrott, 2002; von Borell et al., 2007). Generell ist eine hohe HRV durch eine hohe vagale (parasympathische) Aktivität gekennzeichnet, was auf eine gute physische und psychische Anpassungsfähigkeit hindeutet. Eine verminderte HRV weist somit auf eine Störung des inneren Gleichgewichtes hin (Friedman und Thayer, 1998; Mück-Weymann, 2002). 


\section{Messmethoden und Einflussfaktoren auf die HRV}

Neben den physiologischen Regulationsmechanismen des Organismus gibt es weitere Faktoren, die die HRV beeinflussen und die daher bei der Messung und Interpretation berücksichtigt werden müssen. Zu diesen zählen unter anderem Lebensalter, Geschlecht, Körperlage, Tageszeit, Temperatur, Medikamente, Ausdauerfitness, Stress und Muskelaktivität (Hottenrott, 2002). Diese Einflussfaktoren wurden zwar in Studien am Menschen ermittelt, dennoch wird davon ausgegangen, dass sie auch bei anderen Säugetieren von Bedeutung sind, da die physiologischen Funktionsprinzipien identisch sind (von Borell et al., 2007). Auf Grund der Vielzahl möglicher Einflussfaktoren auf die HRV besteht bei den meisten Parametern (siehe unten) eine sehr weite Spanne möglicher Messergebnisse, die nicht ohne weiteres einem Normalbereich bzw. einer pathologischen Abweichung zugeordnet werden können. Entsprechend sind für die meisten Parameter auch keine Normalwerte definiert (von Borell et al., 2007).

Bei der Messung der HRV wird zwischen Langzeitmessungen (meist 24 Stunden) und Kurzzeitmessungen (meist 5 Minuten) unterschieden. Langzeitmessungen geben Auskunft über die allgemeine Variabilität und können Hinweise auf generelle Disfunktionen in der autonomen Regulation geben (Singer und Ori, 1995). Um spezifische Veränderungen der sympathischen oder parasympathischen Aktivität zu identifizieren sind sie nicht geeignet, da sie einerseits eine Vielzahl an Artefakten aufweisen und andererseits die äußeren Bedingungen (z.B. physische Aktivität) meist nicht genau genug kontrolliert werden können, so dass die Interpretation und Reproduktion der Ergebnisse problematisch ist (Kautzner und Armonk, 1995; Osterhues et al., 1997). Kurzzeitmessungen dagegen liefern Ergebnisse, die denen der Langzeitmessung ähnlich oder sogar besser sind, da die Bedingungen deutlich einfacher zu kontrollieren sind und eine Auswahl artefaktarmer Messbereiche möglich ist (Mooney et al., 1995; Fei et al., 1996; Task Force, 1996). Grundsätzlich können nur Messwerte mit gleicher Messdauer (Kurz- bzw. Langzeitmessung) miteinander verglichen werden. Um chronische Belastungen zu identifizieren, sollten Messbereiche ausgewählt werden, in denen das Tier (bzw. der Mensch) ruhig liegt und 
ungestört ist, um den Effekt von physischer Aktivität oder Erregung möglichst auszuschließen (Langbein et al., 2004; Hagen et al., 2005).

\section{Analysemöglichkeiten und ausgewählte Parameter der HRV}

Die Analyse der HRV kann in drei verschiedenen Bereichen erfolgen.

Die Zeitbereichsanalyse basiert auf den zeitlichen Abständen zwischen den Herzschlägen, wobei noch zwischen aufeinander folgenden Schlägen und allen Schlägen des Messzeitraumes unterschieden wird. Bei der Frequenzbereichsanalyse werden die Anteile von hohen (HF), niedrigen (LF) und sehr niedrigen (very low frequency $=V L F$ ) Frequenzen im Gesamtspektrum des HRV-Signals bestimmt. Die dritte Möglichkeit bietet die geometrische Analyse der HRV-Daten. Hier werden die einzelnen RRIntervalle in ein Streudiagramm (auch Poincaré Plot oder Lorenz Plot genannt) eingetragen. Mit Hilfe orthogonaler Regressionsanalysen werden eine Vertrauensellipse konstruiert und weitere Parameter errechnet (Hottenrott, 2002). Bei jedem Analyseverfahren wird eine Vielzahl von Parametern berechnet. Um Fehlurteile zu vermeiden, sollten bei der Interpretation der HRV immer mindestens zwei Parameter berücksichtigt werden (Hottenrott, 2002). Im Folgenden werden die Parameter SDNN, RMSSD und LF/HF beschrieben, die für Analyse in der vorliegenden Untersuchung herangezogen wurden.

Der Parameter SDNN ist ein Wert der Zeitbereichsanalyse und wird in Millisekunden angegeben. Er ist definiert als die Standardabweichung aller RR-Intervalle im Messzeitbereich. So ist er ein frequenzunabhängiger Indikator für die Gesamtvariabilität.

Der Parameter RMSSD ist ebenfalls ein Wert der Zeitbereichsanalyse und wird in Millisekunden angegeben. Er ist definiert als die Quadratwurzel des quadrierten Mittelwertes der Summe aller Differenzen sukzessiver RRIntervalle. Durch den Fokus auf sukzessive RR-Intervalle gibt dieser Parameter Auskunft über kurzzeitige Veränderungen der RR-Intervalle und repräsentiert somit den parasympathischen Einfluss auf die Herzaktivität.

Der Parameter $\underline{\mathrm{LF} / \mathrm{HF}}$ ist ein Wert der Frequenzbereichsanalyse und beschreibt das Verhältnis zwischen niedrigen und hohen Frequenzanteilen 
am Gesamtsignal. Liegt das Verhältnis in dem Bereich von etwa 1,5 bis 2 wird von sympatho-vagaler Balance gesprochen. Bei Stress (Belastung, Angst usw.) ist die parasympathische Aktivität verringert, was zu erhöhten Werten in diesem Parameter führt (Hottenrott, 2002). 


\section{Beiträge mit Begutachtung}

3.1. Temporary turnout for free exercise in groups: effects on the behaviour of competition horses housed in single stalls

WERHAHN, H.; HESSEL, E.F.; SCHULZE, H.; VAN DEN WEGHE, H.F.A., Journal of Equine Veterinary Science 31 (2011), 417-425. 
3.2. Competition horses housed in single stalls (1st communication): Behaviour and activity patterns during free exercise according to its configuration

WERHAHN, H.; HESSEL, E.F.; VAN DEN WEGHE, H.F.A., Journal of Equine Veterinary Science. Accepted 13.06.2011. 
3.3. Competition horses housed in single stalls (2nd communication): Effects of free exercise on the behaviour in the stable and during training and on the degree of stress

WERHAHN, H.; HESSEL, E.F.; VAN DEN WEGHE, H.F.A.. Journal of Equine Veterinary Science. Accepted, 21.04.2011. 


\section{Diskussion}

\subsection{Bewertung des Versuchsdesigns}

Anders als die klassischen landwirtschaftlichen Nutztiere, lernen Pferde und hier insbesondere die Sportpferde - im Laufe ihres Lebens häufig unterschiedliche Haltungs- und Umgangsformen kennen. Entsprechend sind ihre Erfahrungen sehr individuell, was sich wiederum auf das Verhalten des Einzeltieres auswirkt (Stefanski und Hendrichs, 1996; Houpt, 2005). Um den Einfluss bestimmter individueller Erfahrungen auf das Pferd zu begrenzen, werden als Versuchstiere für wissenschaftliche Untersuchungen häufig junge Pferde (meist bis 3 Jahre) genutzt, die noch nicht gearbeitet werden und ähnlich aufgezogen wurden. Die so gewonnenen Ergebnisse können aber nicht ohne weiteres auf ältere Pferde und speziell auf Sportpferde übertragen werden. Zum einen, weil sich das Verhalten mit fortschreitendem Alter verändert (z.B. weniger Spielverhalten) (Fragen und George, 1977). Zum anderen, weil - zumindest bei Sportpferden - durch das Training und durch die damit meist einher gehende Einzelhaltung (während die Haltung in der Aufzucht häufig eher extensiv und in Gruppen stattfindet) ein deutlich intensiverer Kontakt zum Menschen entsteht, was wiederum starken Einfluss auf das Verhalten hat (Houpt, 2005).

Aus ethologischer Sicht bestehen gerade im Bereich der Sportpferde häufig Defizite in der Haltung, die sowohl fachlich als auch „emotional“ begründet werden (vgl. Kapitel 2.3.1). Daher wurden für die vorliegenden Studien (Kapitel 3.1 bis 3.3) trotz der beschriebenen zu erwartenden Individualität bewusst aktive Sportpferde als Versuchstiere herangezogen.

Praxisnahe Haltung und Training von Sportpferden sind im Versuchsmaßstab nicht möglich, so dass für die Versuche die Kooperation mit Praxisbetrieben notwendig war. Entscheidend für die Auswahl der Betriebe war eine möglichst große Zahl an Warmblutpferden im klassischen Turniereinsatz (Dressur, Springen), die nicht im Verlauf des Versuches den Stall verlassen würden (z.B. Verkauf). Die Pferde mussten weiterhin alle geplanten Versuchsvarianten durchlaufen dürfen (Erlaubnis der Besitzer) und 
vor und während des Versuches von einer Person trainiert werden (um Veränderungen Leistungsbereitschaft dokumentieren zu können). Ein geregelter Tagesablauf (in Bezug auf Fütterungs-, Trainings- und Auslaufzeit) musste mindestens über den Versuchszeitraum eingehalten werden können und ausreichende Auslaufflächen mussten vorhanden sein. Bei der Auswahl der Versuchspferde wurde versucht, Pferde aus Zuchtgebieten mit ähnlichem Zuchtziel, in der Ausbildungsphase und in einem möglichst ähnlichen Alter für die Untersuchungen zu gewinnen.

Die Anzahl an Versuchstieren in den vorliegenden Studien ist im Vergleich zu anderen Untersuchungen dieses Themenbereiches eher gering (Caanitz et al., 1991; Houpt et al., 2001; Chaya et al., 2006; Jørgensen und Bøe, 2007). Dennoch werden die gewonnenen Ergebnisse für aussagekräftig erachtet, da alle Versuchstiere alle Varianten durchliefen und Umwelteinflüsse durch das Versuchsdesign weitgehend als systematische Fehler eingestuft werden konnten. Weiterhin konnte auf diese Weise der Fokus der Auswertung auf die Veränderungen im Verhalten zwischen den Varianten gelegt werden, so dass die absolute Höhe der Werte an Bedeutung verlor. Als Kritikpunkt kann angeführt werden, dass das Training der Pferde nicht standardisiert war. Dieses war nicht möglich, da sich alle Pferde in der Ausbildung befanden und auch während der Versuche im Turniereinsatz waren. Ein Ersatz des regulären durch ein standardisiertes Training hätte zu individuellen Trainingsrückständen und somit letztlich zu einem wirtschaftlichen Schaden des Betriebes geführt. Da die Pferde aber auch während der Versuche im Turniereinsatz waren, wird davon ausgegangen, dass das Training für jedes Pferd mit dem Ziel der individuellen Leistungssteigerung durchgeführt wurde.

Die Dauer einer Variante wurde auf zwei Wochen festgelegt. Eine Verlängerung der Variantendauer war auf Grund der Abhängigkeit von den Betrieben nicht möglich. In früheren Studien aus dem Bereich der Verhaltensforschung bei Pferden wurde ebenfalls mit zwei- oder dreiwöchigen Versuchsvarianten gearbeitet (Sweeting et al., 1986; Jørgensen und Bøe, 2007; Hoffmann, 2008). Es ist möglich, dass diese Versuchsdauer 
nicht ausreicht, um die Pferde an die Varianten zu gewöhnen. Es liegen jedoch keine wissenschaftlichen Erkenntnisse darüber vor, welche Zeit tatsächlich gebraucht wird, um Pferde an einen neuen Tagesrhythmus zu gewöhnen. Die Ergebnisse (Bewegungsaktivität auf der Weide, HRV-Werte der Pferde im Verlauf des Versuches (Kapitel 3.2 und 3.3)) und auch der subjektive Eindruck (z.B. Umgang, Verhalten der Gruppe ohne Auslauf wenn die anderen Pferde auf die Ausläufe verbracht wurden) zeigten, dass die Pferde sich in den beschriebenen Versuchen nach zwei bis drei Tagen an die Varianten gewöhnt hatten. Auch eintägige Unterbrechungen des Tagesrhythmuses (z.B. durch Turniere) hatten kaum Einfluss auf das Verhalten am nächsten Messtag.

Die Abhängigkeit von den Betrieben führte weiterhin dazu, dass der zweite Versuch (Kapitel 3.2 und 3.3) im Hochsommer stattfinden musste. Ein solcher Versuchszeitraum wird als nicht optimal angesehen, da besonders hohe Temperaturen und starke Insektenbelastungen zu erwarten sind, die das Verhalten beeinflussen können (Berger et al., 1999). Jedoch war die Witterung über die angesprochene Versuchszeit sehr konstant, so dass wechselnde Witterungsbedingungen hier keinen entscheidenden Einflussfaktor auf das Verhalten darstellten.

\subsection{Bewertung der Erfassungsmethoden}

Die Auswertung des Verhaltens mit Hilfe digitaler Videoaufzeichnungen und spezieller Auswertungssoftware erwies sich als geeignetes Verfahren. Vorteilhaft waren insbesondere die zeitliche Unabhängigkeit der Auswertung und die Möglichkeit der Variation der Auswertungsgeschwindigkeit. Allerdings muss bei der Vorbereitung darauf geachtet werden, dass auch bei wechselnden Lichtverhältnissen (Sonneneinstrahlung, Dunkelheit) eine ausreichende Aufzeichnungsqualität erreicht wird. Je nach Dauer der Aufzeichnungen fallen sehr große Datenmengen an, was eine entsprechende Leistungsfähigkeit und Speicherkapazität der Computertechnik erfordert. 
Die Methode des Scan Sampling für die direkte Beobachtung der Pferde während des Auslaufs stellte sich als geeignet dar, um die längerfristigen Verhaltensweisen wie Fressen, Dösen, Beschäftigung usw. zu erfassen. Kurzfristige Verhaltensweisen wie z.B. das Wälzen konnten auf dieses Weise nicht sicher erfasst werden, standen aber auch nicht im Fokus der Auswertung. Für die Erfassung der sozialen Interaktionen erschien das Beobachtungsintervall von fünf Minuten teilweise zu lang, da dieses Verhalten auch für kürzere Zeiträume ausgeführt und somit nicht immer sicher erfasst wurde.

Die GPS-Technik zur Erfassung der zurückgelegten Strecke und somit der Bewegungsaktivität zeigte sich als gut geeignet. Die Geräte waren einfach in der Handhabung und die Erfassung sehr zuverlässig. Um Fehleinschätzungen zu vermeiden, muss allerdings im Vorfeld die Messgenauigkeit der Geräte überprüft werden und die Verminderung der Messgenauigkeit in Gebäuden und bei dichter Bewaldung berücksichtigt werden. Auch bei der Positionierung der Geräte am Tier müssen mögliche Fehlinterpretationen durch Bewegungen, die keine Fortbewegung darstellen (z.B. Kopfbewegungen, wenn das Gerät am Halfter befestigt ist), bei der Auswertung berücksichtigt werden.

Bei korrekter Anbringung der Technik am Pferd war die Messung der HRV relativ zuverlässig. Allerdings scheint die Messung bei einigen Tieren besser zu funktionieren als bei anderen, was sich an der Anzahl von Artefakten in den Datensätzen zeigte. Die Methode der Kurzzeitauswertung (5-MinutenIntervalle) ermöglichte es jedoch, Bereiche mit großer Zahl von Artefakten nicht in die Auswertung einzubeziehen, ohne den gesamten Datensatz verwerfen zu müssen. Trotz Polsterung der Messgurte konnten zum Ende des Versuches leichte Hautirritationen am Widerrist einiger Pferde festgestellt werden. Eine Lockerung der Gurte war auf Grund ihrer Beschaffenheit nicht möglich. Außerdem hätte dies zum Verrutschen der Messeinheiten und somit zu einer Unterbrechung der Messung geführt. Eine noch stärkere Polsterung könnte hier möglicherweise Abhilfe schaffen, 
jedoch muss dabei darauf geachtet werden, dass der Kontakt zwischen Körper und Elektroden erhalten bleibt.

Bei der Erfassung der Leistungsbereitschaft muss beachtet werden, dass die Einschätzung durch den Reiter als subjektiv zu bewerten ist. Je nach persönlichem Geschmack und auch individuellem Pferd kann sowohl ein ausgeglicheneres als auch ein „kernigeres“ Pferd als besonders leistungsbereit bewertet werden. Um den subjektiven Einfluss auf die Bewertung zu vermindern, wurde die Leistungsbereitschaft der Pferde in neun Teilbereiche unterteilt (vgl. Kapitel 8.1.2). Bei diesen Teilfragen wurde darauf geachtet, dass möglichst ein konkretes Verhalten (z.B. Widersetzlichkeit, Vorwärtsdrang, Ruhe) erfragt wurde, sodass die persönliche Vorliebe des Reiters für bestimmte Verhaltensweisen die Angaben möglichst nicht beeinflusste. Weiterhin wurden je Pferd nur die Bewertungen eines Reiters berücksichtigt, so dass nicht das absolute Niveau der Werte im Vordergrund der Auswertung stand sondern die Unterschiede zwischen den Varianten. In diesem Zusammenhang wurde außerdem großer Wert darauf gelegt, dass das Verhalten eines Pferdes mit dem sonstigen Verhalten dieses Pferdes im Training verglichen wurde. Unterschiede zwischen verschiedenen Pferden waren für die Untersuchungen nur von untergeordneter Bedeutung. Auf Grund dieser Maßnahmen wird die Art der Erfassung der Leistungsbereitschaft bei nicht standardisiertem Training als geeignet angesehen. Eine zusätzliche Absicherung der Bewertung wäre unter Umständen durch eine zusätzliche Beobachtung des Trainings durch eine weitere Person möglich gewesen. Dieses war in den vorliegenden Versuchen jedoch aus organisatorischen Gründen nicht möglich.

\subsection{Erkenntnisse aus den Managementvarianten über die Tiergerechtheit der Einzelhaltung von Pferden}

Die vorliegenden Untersuchungen zeigen wichtige Aspekte des Managements auf, die die Tiergerechtheit des Systems Einzelhaltung stark beeinflussen. Sowohl Möglichkeiten zur Verbesserung als auch Folgen von suboptimalen Bedingungen werden deutlich. 


\subsubsection{Verhalten in der Box}

Obwohl in den vorliegenden Versuchen keine 24-stündigen Beobachtungen durchgeführt wurden, unterschieden sich die ermittelten Zeitbudgets der Versuchspferde kaum von denen anderer Untersuchungen. Kiley-Worthington (1987) ermittelte bei Pferden in Einzelhaltung mit rationierter Fütterung eine Liegezeit von $15 \%$, was durch die vorliegenden Versuche mit 14 bis $16 \%$ bestätigt werden konnte. Auch die Fressdauer von etwa $15 \%$ konnte mit 14 bis $17 \%$ in den vorliegenden Versuchen bestätigt werden. Somit wurden die verbleibenden 63 bis $66 \%$ mit Stehen verbracht.

Die Versuche haben gezeigt, dass sowohl das Verwehren von freier Bewegung als auch die Gestaltung des Auslaufes das Verhalten der Pferde im Stall (nachmittags und nachts) signifikant beeinflussten. Die vergleichende Auswertung des Verhaltens in den Varianten ergab wichtige Hinweise auf das Wohlbefinden der Pferde.

Die Werte der Liegezeiten lagen insgesamt alle in einem Bereich, der auch in anderen Studien festgestellt wurde (Littlejohn und Munro, 1972; Dallaire, 1986; Kiley-Worthington, 1990; Pedersen et al., 2004). Dennoch weisen einige signifikante Unterschiede auf ein vermindertes Wohlbefinden in bestimmten Varianten hin (Ruckebusch, 1975; Pedersen et al., 2004). Im ersten Versuch (Kapitel 3.1) waren die Unterschiede in der Liegedauer zwischen den Varianten zwar nicht signifikant, dennoch war die Liegezeit am längsten, wenn kein Auslauf gewährt wurde. Im Gegensatz dazu wurde im zweiten Versuch (Kapitel 3.3) die kürzeste Liegezeit beobachtet, wenn kein Auslauf stattfand. Für beide Beobachtungen sind Erklärungsansätze in der Literatur zu finden. Caanitz et al. (1991) begründen eine verlängerte Liegezeit bei mehr körperlicher Aktivität im Tagesverlauf mit einem vermehrten Ruhebedarf als Folge der Aktivität. Laut Fader (2002) kann eine verlängerte Liegezeit auch bei wenig Aktivität im Tagesverlauf als Folge von Langeweile entstehen. Beide Ursachen sind auch in den vorliegenden Versuchen denkbar. Im Hinblick auf die Tiergerechtheit wäre eine verlängerte Liegezeit auf Grund von körperlicher Aktivität wünschenswert.

Es zeigte sich außerdem, dass die Pferde die verkürzte Liegezeit mit mehr Beschäftigung in der Box kompensierten. Neben der Erkundung der 
Boxeneinrichtung (z.B. Trog, Tränke, Gitter) und der Beobachtung der Umgebung wurde auch die Erkundung und Aufnahme der Stroheinstreu als Beschäftigung dokumentiert. Für die Pferde wird durch die Aufnahme von Stroh die Fresszeit verlängert, was dem natürlichen Verhalten des Pferdes, viel Zeit mit der Futteraufnahme zu verbringen, entgegen kommt (Cooper und Albentosa, 2005; Houpt, 2005). Mit der Nutzung von Stroh als Einstreu kann also das Risiko von Langeweile und Frustration und damit der Entwicklung von Stereotypien gemindert werden (Malpass und Weigler, 1994; McGreevy et al., 1995; McGreevy et al., 1995; Winskill et al., 1996). Die Aufnahme von Stroh kann durch den hohen Rohfaseranteil auch die Verdauungstätigkeit fördern. Wobei zu große Aufnahmemengen wiederum zu Problemen im Verdauungstrakt führen können, z.B. in Form von Verstopfungen (Meyer und Coenen, 2002).

Im ersten Versuch (Kapitel 3.1) wurden in der Variante ohne Auslauf eine deutliche Unruhe und auch eine erhöhte Aggressivität gegenüber den Nachbarpferden festgestellt, was als vermindertes Wohlbefinden der Tiere interpretiert wurde. Unruhe oder Aggressivität konnten an Hand der Verhaltensbeobachtungen im zweiten Versuch (Kapitel 3.3) nicht bestätigt werden. Die Ermittlung der Herzfrequenzvariabilität ergab hier jedoch eindeutig die höchste Stressbelastung in der Variante ohne Auslauf. Obwohl im ersten Versuch die Herzfrequenzvariabilität nicht erfasst werden konnte, lassen die Ergebnisse vermuten, dass das Verhalten wertvolle Hinweise auf eine Stressbelastung geben kann. Im zweiten Versuch zeigte sich allerdings, dass ein unauffälliges Verhalten nicht zwingend die Abwesenheit von Stress bedeutet, was auch von Mason und Latham (2004) zu bedenken gegeben wird. Entsprechend sollten für eine möglichst sichere Beurteilung der Stressbelastung daher mehrere Parameter (z.B. Verhalten, HRV, Cortisolbestimmung) herangezogen werden (Cooper und Mason, 1998; Henderson, 2007; Hoffmann, 2008).

Die Gestaltung des Auslaufs beeinflusste das Verhalten in der Box kaum. Signifikante Unterschiede zeigten sich nur in der Frequenz des Dösens (häufiger bei Auslauf vor dem Training als bei Auslauf nach dem Training) 
und des Liegens (häufiger bei Gruppenauslauf als bei Einzelauslauf). Die vorliegenden Daten erlauben keine genaue Interpretation dieser Beobachtungen. Allein an Hand der Verhaltensbeobachtungen in der Box lassen sich bei diesen Versuchsanstellungen daher keine Vor- oder Nachteile der untersuchten Auslaufvarianten feststellen.

\subsubsection{Verhalten und Bewegungsaktivität im Auslauf}

Bei dem Wechsel von einer Variante ohne Auslauf zu einer Variante mit Auslauf zeigten die Pferde in beiden Versuchen (Kapitel 3.1 und 3.2) eine erhöhte Bewegungsaktivität am ersten Auslauftag. Innerhalb der Varianten mit Auslauf und auch bei dem Wechsel zu einer anderen Variante mit Auslauf verhielten sich die Pferde deutlich ruhiger. Bei regelmäßiger freier Bewegung kann folglich von einer geringen Verletzungsgefahr im Zusammenhang mit der Bewegungsaktivität im Auslauf ausgegangen werden. Dass gerade unregelmäßige freie Bewegung $z u$ einer hohen Bewegungsaktivität während des Auslaufes führt, wird auch von früheren Untersuchungen berichtet (Dantzer et al., 1983; Dellmeier et al., 1985; Hogan et al., 1988; Mal et al., 1991; Houpt et al., 2001; Chaya et al., 2006). Dennoch ist die Angst vor intensiver Bewegungsaktivität insbesondere zu Beginn des Auslaufs und dem daraus resultierenden Verletzungsrisiko (Kapitel 2.3.2) noch heute einer der Hauptgründe für das nicht Gewähren von freier Bewegung.

Um zu verhindern, dass Pferde sich während des Auslaufes gegenseitig verletzen, wird freie Bewegung häufig nur einzeln angeboten. Diese Form des Managements ist im Hinblick auf die Erfüllung des Bewegungsbedarfes des Pferdes sicherlich als besser einzuschätzen als ein vollständiger Verzicht auf freie Bewegung. Dennoch bleibt bei der Haltung in klassischen hoch vergitterten Einzelboxen die Problematik der fehlenden Sozialkontakte (Kapitel 2.1.3 und 2.2.5). Um vielen Pferden Einzelauslauf zu ermöglichen, benötigt ein Betrieb außerdem entweder sehr viele Auslaufflächen oder die Pferde müssen häufig gewechselt werden, was einen hohen Zeitaufwand mit sich bringt. Die vorliegenden Untersuchungen haben gezeigt, dass das Verhalten der Pferde ruhiger war (insgesamt weniger zurückgelegte Strecke, 
deutlich weniger Aktivitätsspitzen), wenn der Auslauf in Zweiergruppen stattfand. Mögliche Gründe für diese Beobachtung sind in Kapitel 3.2 ausführlich diskutiert. Eine entscheidende Voraussetzung für einen weitgehend gefahrlosen gemeinsamen Auslauf ist allerdings die Verträglichkeit der Pferde. Da Pferde enge Freundschaften ausbilden, wenn sie die Möglichkeit dazu bekommen, sollten möglichst feste Auslaufpartnerschaften etabliert werden (Tyler, 1972; Clutton-Brock et al., 1976; Duncan und Cowtan, 1980; McDonnell, 2002; Broom und Fraser, 2007). Dieses wurde in den dargestellten Versuchen berücksichtigt und es wurden auch keine aggressiven sozialen Interaktionen zwischen den Auslaufpartnern beobachtet. Bei Auslauf in stabilen Gruppen kann somit sowohl das Bedürfnis nach Bewegung als auch nach sozialer Interaktion erfüllt werden, was die Tiergerechtheit der Haltungsform entscheidend verbessert.

Der erste Versuch ergab eine signifikant kürzere im Auslauf zurückgelegte Strecke, wenn die Pferde vorher trainiert wurden. Entsprechend wurde die Verletzungsgefahr in dieser Variante geringer eingeschätzt, als wenn der Auslauf vor dem Training stattfand. Zum einen wird dieses durch die insgesamt geringere Bewegungsaktivität begründet. Zum anderen waren die Pferde durch das Training bereits kontrolliert „aufgewärmt“ worden, so dass der Bewegungsapparat elastisch und in der Folge weniger anfällig für Verletzungen war (Kusunose et al., 1987; Safran et al., 1988). Auf Grund dieser Erkenntnisse wurde der Auslauf auch im dritten Versuch nach dem Training angeboten.

Die Aussage, dass unstrukturierte Ausläufe (hier: ohne Trennung von Funktionsbereichen, ohne Futterangebot zusätzlich zu möglichem Bewuchs, ohne Tränke usw.) keine ausreichenden Bewegungsanreize schaffen und somit zu keiner nennenswerten Bewegungsaktivität seitens der Pferde führen (Zeitler-Feicht, 2008), kann durch die vorliegenden Versuche nicht bestätigt werden. Die Messungen in beiden Versuchen (Kapitel 3.1 und 3.2) ergaben bei zweistündiger Beobachtungszeit in allen Varianten zwischen zwei und drei zurückgelegte Kilometer im unstrukturierten Auslauf. Dieses Ergebnis widerspricht außerdem der These, dass der Bewegungsbedarf durch das 
Training gedeckt wird. Dass auch nach dem Training noch Bewegungsbedarf besteht, wird auch von Jørgensen und Bøe (2007) bestätigt.

Im zweiten Versuch (Kapitel 3.2) zeigte sich, dass bei der Wahl der Weide als Auslaufform der Großteil der zweistündigen Auslaufzeit mit Fressen verbracht wurde. Diese Verhaltensweise ist aus physiologischer und ethologischer Sicht als positiv zu bewerten (vgl. Kapitel 2.1.1 und 2.2.2). Eine bisher nicht veröffentlichte Untersuchung (Werhahn et al., in press) hat außerdem gezeigt, dass die Bewegungsaktivität bei Auslauf auf der Weide in Folge der Beschäftigung mit dem Fressen signifikant geringer ist, als wenn der Auslauf auf einer ebenso großen unbewachsenen Fläche stattfindet. Entsprechend wird auch das Risiko für Verletzungen bei Weidegang geringer eingeschätzt, da das Fressen anderen Verhaltensweisen offenbar vorgezogen wird (zumindest bei der untersuchten Auslaufdauer von zwei bzw. drei Stunden). Bei deutlich längerer Auslaufzeit muss allerdings je nach Bewuchs und Prädisposition des individuellen Pferdes im Hinblick auf Gesundheit und Leistungsfähigkeit eine Nährstoffüberversorgung durch die Aufnahme von Gras vermieden werden (Meyer und Coenen, 2002; Ellis, 2003).

Forschungsbedarf besteht in diesem Zusammenhang im Hinblick auf die optimale Auslaufdauer bei der Einzelhaltung von Sportpferden. Aus Sicht der Ethologie ist eine möglichst lange Auslaufzeit erstrebenswert (Kapitel 2.2). Bisher konnte jedoch lediglich wissenschaftlich festgestellt werden, dass die Gruppenauslaufhaltung (fünf Pferde) im Vergleich zur Einzelboxenhaltung (mit täglich einstündigem Auslauf) positive Auswirkungen auf die Ausdauerleistungsfähigkeit hat (Arnemann, 2003). Für die Einzelhaltung liegen bisher keine Erkenntnisse über die Auswirkungen unterschiedlicher Auslaufdauern auf die Leistungsfähigkeit und/oder die Leistungsbereitschaft vor.

\subsubsection{Stressbelastung}

Auf Grund der technischen Probleme bei der nächtlichen Aufzeichnung der Herzfrequenzdaten im ersten Versuch konnte die Stressbelastung der Pferde in diesem Versuch nicht ausgewertet werden. Die Pferde zeigten jedoch in der Variante ohne Auslauf in diesem Versuch eine signifikant größere Unruhe und Aggressivität gegenüber den Nachbarpferden im Stall, was laut 
Mal et al. (1991) als Ausdruck einer erhöhten Stressbelastung interpretiert werden kann.

Im zweiten Versuch unterschied sich das Verhalten im Stall in der Variante ohne Auslauf zwar kaum von den anderen Varianten, an Hand der HRV war jedoch im Durchschnitt über die Pferde eine deutlich höhere Stressbelastung zu erkennen, wenn kein Auslauf angeboten wurde. Die teilweise sehr große Varianz zwischen den einzelnen Pferden erschwerte die Interpretation der Ergebnisse der Individuen. Auch in der Literatur wird von großen Streuungen der HRV-Parameter SDNN und RMSSD berichtet (Voss et al., 2002; Rietmann et al., 2004; Norman et al., 2005), wobei die Pferde im vorliegenden Versuch nicht alle Pferde in der Variante ohne Auslauf die geringsten HRV-Werte zeigten. Laut Henderson (2007) sind die Anfälligkeit gegenüber chronischem Stress, die notwendige „Einwirkzeit“ des Stresses bis er messbar ist und auch die Art und Weise der Reaktion auf die Belastung sehr stark vom Individuum abhängig. Die Darstellung der mittleren HRV-Werte an den einzelnen Messtagen (Kapitel 3.3, Figure 5) erweckt den Eindruck, dass die Werte ab der zweiten Woche der Varianten relativ konstant bleiben. Aus der Literatur geht nicht hervor, wie lange es dauert, bis eine chronische Stressbelastung messbar ist. Möglicherweise wäre eine Verlängerung der Varianten hilfreich gewesen, um die gewonnenen Ergebnisse abzusichern.

\subsubsection{Leistungsbereitschaft}

Die Ergebnisse aus beiden Versuchen zeigen eindeutig, dass die untersuchten Auslaufvarianten keinen negativen Einfluss auf die Leistungsbereitschaft der Pferde hatten. Die Unterschiede zwischen den Varianten waren zwar nicht signifikant, jedoch zeigte sich eher eine positive Auswirkung des Auslaufes auf die Beurteilung der Leistungsbereitschaft. Das Argument, dass Sportpferden bei freier Bewegung die Motivation zur sportlichen Leistung verloren geht, kann für die untersuchten Varianten somit nicht bestätigt werden. Arnemann (2003) konnte zusätzlich nachweisen, dass die Gruppenauslaufhaltung im vergleich zur Einzelboxenhaltung (mit täglich 
einer Stunde Auslauf) positive Auswirkungen auf die Ausdauerleistungsfähigkeit von Pferde hat.

Die These, dass der Bewegungsbedarf der Pferde durch das Training gedeckt wird, kann ebenfalls nicht bestätigt werden. In allen Auslaufvarianten wurde auch an Tagen mit sehr ruhigem Verhalten im Auslauf mindestens ein Kilometer zurückgelegt. Fand der Auslauf nach dem Training statt, war die zurückgelegte Strecke zwar kürzer als wenn er vor dem Training stattfand, da jedoch noch immer Bewegung zu verzeichnen war, wird davon ausgegangen, dass noch Bewegungsbedarf bestand. Diese Beobachtung wird auch von Jørgensen und Bøe (2007) bestätigt. Da die Versuche unter Praxisbedingungen stattfanden, kann nicht geklärt werden, ob die gezeigte Bewegung auf endogene (inneres Bedürfnis nach Bewegung) oder exogene Faktoren (äußere Einflüsse wie z.B. Bewegung anderer Pferde, Suche nach Futter, Störung durch Insekten, Unruhe in der Umgebung) zurück zu führen war. Ebenfalls ungeklärt ist, ob ein späterer Auslaufzeitpunkt beispielsweise am Abend (bei Training am Vormittag) zu einer ähnlich hohen Bewegungsaktivität im Auslauf geführt hätte wie in der Variante Auslauf vor dem Training, da sich möglicherweise im Laufe des Aufenthaltes im Stall erneut Bewegungsbedarf „aufbauen“ könnte. Die Klärung der Bedeutung dieser Faktoren (endogen und exogen begründete Bewegung) im Zusammenhang mit dem optimalen Auslaufzeitpunkt bedarf weiterer Forschung. Kein Zusammenhang konnte zwischen der im Training zurückgelegten Strecke und der Strecke des im Anschluss stattfindenden Auslaufs festgestellt werden. Die Trainingsintensität hatte in diesen Versuchen also offensichtlich keinen Einfluss auf die Bewegungsaktivität im Auslauf. 


\section{Zusammenfassung}

Aus ethologischer Sicht ist die Tiergerechtheit der Einzelboxenhaltung von Pferden kritisch zu bewerten. Ohne freie Bewegungsmöglichkeiten wird diese Haltungsform in der Wissenschaft als nicht tiergerecht angesehen. Dennoch werden in der Praxis viele Pferde in diesem System gehalten. Im Bereich der Sportpferde ist diese Haltungsform fast ausschließlich vertreten, wobei den Tieren aus unterschiedlichen Gründen häufig keine freien Bewegungsmöglichkeiten angeboten werden.

Ziel der vorliegenden Arbeit war es, die Auswirkungen unterschiedlicher Managementvarianten auf das Verhalten von Sportpferden in mehreren Bereichen systematisch zu untersuchen. In zwei unabhängigen Versuchsanstellungen wurde der Einfluss des Auslaufzeitpunktes und der Auslaufgestaltung (Einzelauflauf/Gruppenauslauf) analysiert. An Hand der Ergebnisse wurden die Varianten im Hinblick auf inren Einfluss auf die Tiergerechtheit und die Eignung für die Praxis beurteilt.

Im Mittelpunkt des ersten Versuches standen die Effekte der Varianten (a) Auslauf vor dem Training, (b) Auslauf nach dem Training und (c) kein Auslauf zusätzlich zum Training auf das Verhalten von vier Sportpferden in Einzelboxenhaltung. Das Verhalten der Pferde in der Box wurde mit Hilfe von Videoaufzeichnungen zwischen 12.30 und $7.30 \mathrm{Uhr}$ an drei Tagen jeweils am Ende der Varianten kontinuierlich ausgewertet. Das Verhalten während des zweistündigen Auslaufs auf einer unbewachsenen Fläche wurde direkt beobachtet und die dabei zurückgelegte Strecke mit Hilfe von GPS-Geräten erfasst. Das Verhalten der Pferde im Training wurde an Hand eines Fragebogens von den Reitern dokumentiert. In der Variante ohne Auslauf war das Verhalten der Pferde im Stall unruhiger als in den anderen Varianten, was sich an häufigeren Wechseln zwischen den Verhaltensweisen zeigte. Die GPS-Messungen ergaben eine signifikant kürzere zurückgelegte Strecke im Auslauf, wenn die Pferde vorher trainiert wurden. In dieser Variante zeigten sie außerdem weniger Trab und Galopp und dafür mehr Dösen. Das Verhalten der Pferde im Training unterschied sich nicht signifikant zwischen den Varianten, so dass kein negativer Einfluss der freien 
Bewegung auf die Leistungsbereitschaft der Pferde festgestellt werden konnte. Da davon ausgegangen wird, dass eine geringere Bewegungsaktivität auch eine geringere Verletzungsgefahr mit sich bringt, wird Auslauf nach dem Training als die beste der untersuchten Varianten angesehen.

Der zweite Versuch beschäftigte sich mit den Auswirkungen der Varianten (a) Weidegang einzeln nach dem Training, (b) Weidegang in Zweiergruppen nach dem Training und (c) kein Auslauf zusätzlich zum Training auf das Verhalten und die Stressbelastung von sechs Sportpferden in Einzelhaltung. Das Verhalten der Pferde während des zweistündigen Weideganges wurde direkt beobachtet und die zurückgelegte Strecke mit Hilfe von GPS-Geräten erfasst. Das Verhalten der Pferde in der Box wurde an Hand von Videoaufzeichnungen zwischen 14.00 und 6.00 Uhr an drei Tagen jeweils am Ende der Varianten kontinuierlich ausgewertet. Die Stressbelastung wurde mit Hilfe der Herzfrequenzvariabilität und zwar durch die Analyse der Parameter SDNN, RMSSD und LF/HF bestimmt. Das Verhalten der Pferde im Training wurde von den Reitern unter Verwendung eines Fragebogens erfasst. Die Beobachtung des Verhaltens der Pferde während des Weideganges ergab, dass die meiste Zeit des Auslaufes mit Fressen verbracht wurde. Erst nach etwa 90 Minuten wurde das Fressen vermehrt durch Beschäftigung, Bewegung und soziale Interaktionen abgelöst. Bei Gruppenauslauf wurden mehr soziale Interaktionen und weniger Bewegung festgestellt als bei Einzelauslauf. Entsprechend war auch die zurückgelegte Strecke bei Einzelauslauf signifikant länger. Das Verhalten der Pferde im Stall ergab eine signifikant kürzere Liegedauer und entsprechend eine längere Beschäftigungsdauer in der Variante ohne Weidegang im Vergleich zu Gruppenauslauf. Die Messung der Herzfrequenzvariabilität ergab zwar große individuelle Unterschiede, insgesamt war jedoch die Stressbelastung in der Variante ohne Auslauf größer als wenn Weidegang angeboten wurde. Das Verhalten der Pferde im Training wurde in den Varianten mit Weidegang etwas besser beurteilt als in der Variante ohne Auslauf. Der Weidegang in Zweiergruppen ist im Hinblick auf die Bedürfnisse des Pferdes und die 
Ansprüche des Reiters von den untersuchten Varianten eindeutig am besten zu beurteilen.

Insgesamt zeigen die Untersuchungen, dass das Auslaufmanagement das Verhalten und die Stressbelastung der Pferde sowohl am Tag als auch in der Nacht beeinflusst. Die Zeit im Auslauf wird sowohl für Bewegung als auch für Sozialkontakte mit Artgenossen genutzt und führt zu einer Minderung der Stressbelastung im Vergleich zu einem Verwehren von freier Bewegung. Im Sinne der Tiergerechtheit sollte die Stressbelastung in jedem Haltungssystem minimiert werden. Die vorliegende Arbeit zeigt eindeutig den positiven Effekt des Auslaufes in Gruppen auf das Wohlbefinden von Sportpferden in Einzelboxenhaltung, wobei keine negativen Auswirkungen auf die Leistungsbereitschaft festgestellt werden konnten. 


\section{Summary}

\section{Variations in the management of turnout in competition horses housed in single stalls: evaluation of the effects on animal welfare with the aid of animal behaviour and the degree of stress}

From an ethological point of view, the animal welfare in single housing systems must be evaluated critically. Without free exercise, scientists consider this system as not being suitable for horses, despite its widespread use. Particularly competition horses are almost exclusively kept in this type of housing and the provision of free exercise is often denied for various reasons.

The aim of the experiments in this study was a systematic investigation of the effects of different management practices on the behaviour of competition horses in several respects. In two independent experiments, the influence of the particular time of turnout and the turnout configuration (solitary/group) was analysed. The treatments were evaluated with regard to their effects on animal welfare and their suitability for practical use.

In the first experiment, the effects of the treatments (a) turnout before training, (b) turnout after training and (c) no turnout on the behaviour of four competition horses housed in single stalls were analysed. The horses were concomitantly subjected to their normal training regime. The behaviour of the horses in the stable was continuously analysed via video recordings taken between $12.30 \mathrm{pm}$ and $7.30 \mathrm{am}$ on three days at the end of each treatment period. The behaviour during turnout (two hours) on a grassless paddock was observed directly and the distance covered was measured by GPS devices. The horses' behaviour during training was documented by the riders with the aid of a questionnaire. In the treatment without turnout, the behaviour in the stable was more restless than in the other two treatments. This restlessness was indicated by more frequent changes between the individual behaviour patterns. The GPS measurements showed that the horses covered a significantly shorter distance during turnout when they were trained beforehand. In this treatment, the horses also showed less trotting 
and cantering and more dozing during turnout. The behaviour during training did not differ significantly between the treatments, so the willingness to perform was not negatively influenced by free exercise. Turnout after training is considered to be the best of the analysed treatments as the horses behaved more quiet in the stable and less activity during turnout is associated with a lower risk of injury.

The second experiment dealt with the effects of the treatments (a) solitary turnout on pasture after training, (b) turnout on pasture in groups of two after training and (c) no turnout in addition to training on the behaviour and degree of stress in six competition horses housed in single stalls. The pasture was covered with grass. The behaviour of the horses during the two-hour turnout period was observed directly and the distance covered was measured by GPS devices. The behaviour of the horses in the stable was continuously analysed with the aid of video recordings between $2 \mathrm{pm}$ and 6 am on three days at the end of each treatment. The degree of stress was analysed via heart rate variability using the parameters SDNN, RMSSD and LF/HF. The horses' behaviour during training was documented by the riders with the aid of a questionnaire. The direct observation during turnout showed that most of the time was spent eating. Only after 90 minutes was eating increasingly replaced by occupation, exercise and social interaction. In group turnout more social interaction and less exercise was observed than in solitary turnout. Accordingly, the distance covered was significantly greater in solitary turnout. The behaviour of the horses in the stable in the treatment without turnout involved significantly less lying down and a concomitant increase in occupation compared to the turnout in groups. Though the analysis of heart rate variability revealed great individual differences, the greatest degree of stress was found in the treatment without turnout. The behaviour during training was evaluated as being slightly better in the treatments with turnout than without. Regarding the requirements of both the horse and the rider, the treatment turnout in groups is considered as being the most suitable of the analysed treatments. 
In conclusion, the study has demonstrated that the management of turnout affects horse behaviour and the degree of stress not only during the day but also during the night. Standard animal welfare practices dictate that the amount of stress imposed on animals should be minimised. When turnout was allowed, the horses used it for exercise and social interaction, and the degree of stress was decreased compared to when turnout was denied, showing that turnout is able to improve animal welfare. The present study clearly shows the positive effect of turnout in groups on the welfare of competition horses housed in single stalls, while no negative effects on their willingness to perform were found. 


\section{Literaturverzeichnis}

Ahlswede L, Bottermann H, Düe M, Müller C, Vogel M (2005). Eckdaten Pferd. Heft 1: Haltung. FN-Verlag, Warendorf.

Arnemann S (2003). Haltung von Sportpferden unter besonderer Berücksichtigung der Leistung. Dissertation, Tierärztliche Hochschule Hannover.

Bahr C (2007). Sensorbasierte Analyse und Modellierung ausgewählter Verhaltensparameter von Mutterkühen. Dissertation, Humboldt Universität zu Berlin.

Bender I (1999). Praxishandbuch Pferdehaltung. Franck-Kosmos Verlags$\mathrm{GmbH} \&$ Co., Stuttgart.

Berger A, Scheibe KM, Eichhorn K, Scheibe A, Streich J (1999). Diurnal and ultradian rhythms of behaviour in a mare group of Przewalski horse (Equus freus przewalskii), measured through one year under semi-reserve conditions. Appl Anim Behav Sci, 64, 1-17.

BMELV (2009). Leitlinien zur Beurteilung von Pferdehaltungen unter Tierschutzgesichtspunkten. Herausgegeben vom Herausgegeben vom Bundesministerium für Ernährung Landwirtschaft und Verbraucherschutz, http://www.bmelv.de/SharedDocs/Downloads/Landwirtschaft/Tier/Tierschutz/ GutachtenLeitlinien/HaltungPferde.pdf? blob=publicationFile (Abrufdatum: 15.05.2010).

Borstel UKv, Kassebaum L, Ladewig K, Gauly M (2010). Arbeitszeitaufwand in der Pferdehaltung: ein Vergleich von Einzelboxen-, Laufstall- und Bewegungsstallhaltung. Züchtungskunde, 82, 417-427.

Boyd L, Bandi N (2002). Reintroduction of takhi, Equus ferus przewalskii, to Hustai National Park, Mongolia: time budget and synchrony of activity preand post-release. Appl Anim Behav Sci, 78, 87-102.

Boyd LE, Carbonaro DA, Houpt KA (1988). The 24-hour time budget of Przewalski horses. Applied Animal Behaviour Science, 21, 1-2, 5-17.

Bracher VD (1987). Zur Erfassung der Dunkelziffer COPD-kranker Pferde. Dissertation, Universität Zürich.

Brade W (2001). Tiergerechte Milchrinderhaltung - Definition, Anforderungen und Kriterien. Praktischer Tierarzt, 82, 588-594.

Brehme U, Stollberg U, Holz R, Schleusener T (2008). ALT pedometer - New sensor-aided measurement system for improvement in oestrus detection. Comput Electron Agr, 62, 73-80.

Broom DM (1991). Animal welfare: concepts and measurement. J Anim Sci, 69, 4167-4175.

Broom DM, Fraser AF (2007). Domestic animal behaviour and welfare. CAB International, Wallingford. ISBN: 987-1-84593-287-9.

Butler I, Armbruster B (1984). Struktur und Abgangsursachen bei Schlachtpferden. Dtsch tierärztl Wschr, 91, 330-331. 
Caanitz H, O'Leary L, Houpt KA, Petersson K, Hintz H (1991). Effect of exercise on equine behavior. Appl Anim Behav Sci, 31, 1-12.

Chaya L, Cowan E, McGuire B (2006). A note on the relationship between time spent in turnout and behaviour during turnout in horses (Equus caballus). Appl Anim Behav Sci, 98, 155-160.

Clausen M, Preisinger R, Kalm E (1990). Analyse von Krankheitsdaten in der deutschen Warmblutzucht. Züchtungskunde, 62, 167-178.

Clutton-Brock TH, Greenwood PJ, Powell RP (1976). Ranks and relationships in Highland ponies and Highland cows. Z Tierpsychol, 41, 202-216.

Cooper JJ, Albentosa MJ (2005). Behavioural adaptation in the domestic horse: potential role of apparently abnormal responses including stereotypic behaviour. Livest Prod Sci, 92, 2, 177-182.

Cooper JJ, Mason GJ (1998). The identification of abnormal behaviour and behavioural problems in stabled horses and their relationship to horse welfare: a comparative review. Equine Vet J, 27, 5-9.

Dallaire A (1986). Rest behavior. Equine Pract, 2, 591-607.

Dallaire A (1993). Stress and behavior in domestic animals: Temperament as a predisposing factor to stereotypies. Annals of the New York Academy of Sciences, 697, 269-274.

Dantzer R, Mormede T, Bluthe RM, Soissons J (1983). The effect of different housing conditions on behavioral and adrenocortical reactions in veal calves. Reprod Nutr Develop, 23, 501-508.

de Jong IC, Sgoifo A, Lambooij E, Korte SM, Blokhuis HJ, Koolhaas JM (2000). Effects of social stress on heart rate and heart rate variability in growing pigs. Can J Anim Sci, 80, 273-280.

Dellmeier GR, Friend TH, Gbur EE (1985). Comparison of four methods of calf confinement. II. Behavior. J Anim Sci, 60, 1102-1109.

Désiré L, Veissier I, Desprès G, Boissy A (2004). On the way to assess emotions in animals: do lambs (Ovis aries) evaluate an event through its suddenness, novelty, or unpredictability? J Comp Psychol, 118, 363-74.

Douglas JE, Mittal C, Thomason JJ, Jofriet JC (1996). The modulus of elasticity of equine hoof wall: implications for the mechanical function of the hoof. J Exp Biol, 199, 1829-1836.

Duncan P (1980). Time-budgets of Camargue horses. Behaviour, 72, 26-49.

Duncan P, Cowtan P (1980). An unusual choice of habitat helps Camargue horses to avoid blood-sucking horse-flies. Biol Behav, 5, 55-60.

Elia JB (2002). The effects of diets differing in fiber content on equine behavior and motivation for fiber. Cornell University, New York.

Elia JB, Erb HN, Houpt KA (2010). Motivation for hay: Effects of a pelleted diet on behavior and physiology of horses. Physiology and Behavior, 101, 623-627.

Ellis AD (2003). Ingestive and digestive processes in the horse. PhD thesis, Writtle College, Essex University. 
Elsässer F, Klobasa F, Ellendorff F (2001). ACTH Stimulationstest und Bestimmung von Cortisol in Blut und Speichel zur Bewertung des Trainingszustands / der Kondition beim Warmblutpferd. Dtsch Tierarztl Wochenschr, 108, 31-36.

Fader C (2002). Ausscheide- und Ruheverhalten von Pferden in Offenlaufstall- und Boxenhaltung. Dissertation, Technische Universität München.

Fei L, Copie X, Malik M, Camm AJ (1996). Short and long-term assessment of heart rate variability for risk stratification after acute myocardial infarction. Am J Cardiol, 77, 681-684.

Fragen RM, George TK (1977). Play behaviour and exercise in young ponies (Equus caballus). Behav Ecol Sociobiol, 2, 267-269.

Fraser AF, Broom DM (2004). Farm animal behaviour and welfare. 9. korrigierte Ausgabe, Verlag CABI Publishing, Bath.

Fraser D (2008). Understanding animal welfare. Acta Veterinaria Scandinavica, 50, 1-7.

Frentzen F (1994). Bewegungsaktivitäten und -verhalten von Pferden in Abhängigkeit von Aufstallungsform und Fütterungsrhythmus unter besonderer Berücksichtigung unterschiedlich gestalteter Auslaufsysteme. Dissertation, Tierärztliche Hochschule Hannover.

Friedman BH, Thayer JF (1998). Anxiety and autonomic flexibility: a cardiovascular approach. Biol Psychol, 47, 243-263.

Ganskopp D (2001). Manipulating cattle distribution with salt and water in large arid-land pastures: a GPS/GIS assessment. Appl Anim Behav Sci, 73, 251- 262.

Gattermann R (Hrsg.) (2006). Wörterbuch zur Verhaltensbiologie der Tiere und des Menschen. 2. Auflage, Elsevier, Spektrum Akademischer Verlag, ISBN: 9783827417039.

Gattermann R (Hrsg.) ( 2006). Wörterbuch zur Verhaltensbiologie der Tiere und des Menschen. 2. Auflage, Elsevier, Spektrum Akademischer Verlag, 9783827417039.

Gehr P, Erni H (1980). Morphometric estimation of pulmonary diffusion capacity in two horse lungs. Respiration Physiology, 41, 199-210.

Gehr P, Mwangi DK, Ammann A, Maloiy GMO, Richard Taylor C, Weibel ER (1981). Design of the mammalian respiratory system. V. Scaling morphometric pulmonary diffusing capacity to body mass: Wild and domestic mammals. Respiration Physiology, 44, 61-86.

Gerber V, Robinson NE, Luethi S, Marti E, Wampfler B, Straub R (2003). Airway inflammation and mucus in two age groups of asymptomatic wellperforming sport horses. Equine Vet J, 35, 5, 491-495.

Geverink NA, Schouten EG, Gort G, Wiegant VM (2002). Individual differences in behavioral and physiological responses to restraint stress in pigs. Physiol Behav, 77, 451-457. 
Goodwin D, Davidson HPB, Harris P (2002). Foraging enrichment for stabled horses: effects on behaviour and selection. Equine Vet J, 34, 686-691.

Hagen K, Langbein J, Schmied C, Lexer D, Waiblinger S (2005). Heart rate variability in dairy cows--influences of breed and milking system. Physiol Behav, 85, 195-204.

Haidn B, Berger N, Gruber V, Lindenau G (2002). Arbeitszeitbedarf für die Pensionspferdehaltung in landwirtschaftlichen Betrieben. KTBLSonderveröffentlichung Nr. 041, KTBL-Schriften-Vertrieb im Landwirtschaftsverlag $\mathrm{GmbH}$, Münster.

Hale LA, Huggins SE (1980). The electroencephalogram of normal 'grade' pony in sleep and wakefulness. Comp Biochem Physiol, 66A, 251-257.

Hebenbrock M (2005). GPS gestütztes Monitoring von Hochleistungsvielseitigkeitspferden in Wettkampf und Training. Dissertation, Tierärztliche Hochschule Hannover.

Henderson AJZ (2007). Don't Fence Me In: Managing Psychological Well Being for Elite Performance Horses. J Appl Anim Welfare Sci, 10, 4, 309 329.

Hoffmann G (2008). Bewegungsaktivität und Stressbelastung bei Pferden in Auslaufhaltungssystemen mit verschiedenen Bewegungsangeboten. Dissertation, Universität Gießen.

Hogan ES, Houpt KA, Sweeney K (1988). The effect of enclosure size on social interactions and daily activity patterns of the captive Asiatic wild horse (Equus przewalskii). Appl Anim Behav Sci, 21, 147-168.

Holcombe SJ, Jackson C, Gerber V, Jefcoat A, Berney C, Eberhardt S et al. (2001). Stabling is associated with airway inflammation in young Arabian horses. Equine Vet J, 33, 244-249.

Hommerich G (1995). Ausfallursachen und -frequenzen. In: Tagungsband Göttinger Pferdetage 1995 - Zucht und Haltung von Sportpferden, 127-132.

Hottenrott K (2002). Herzfrequenzvariabilität im Sport. Czwalina Verlag, Schriften der Deutschen Vereinigung für Sportwissenschaften Band 129, Hamburg, Germany.

Hottenrott K (2002). Herzfrequenzvariabilität im Sport [Heart rate variability in sport]. Czwalina Verlag, Schriften der Deutschen Vereinigung für Sportwissenschaften Band 129, Hamburg, Germany.

Houpt KA (2005). Domestic Animal Behaviour for Veterinarians and Animal Scientists. Blackwell Publishing Professional, Ames, lowa, USA. ISBN: 08138-0334-9.

Houpt KA, Houpt TR, Johnson JL, Erb HN, Yeson SC (2001). The effects of exercise deprivation on the behavior and physiology of straight stall confined pregnant mares. Anim Welfare, 10, 257-267.

Houpt KA, Kusunose R (2000). Genetics of behaviour. In: Bowling, A.T.; Ruvinsky, A. (ed.): The genetics of the horse. S. 281-306, CABI Publishing, Wallingford. ISBN: 0-85199-429-6. 
Houpt KA, McDonnell SM (1993). Equine stereotypies. Comp Cont Educ Pract Vet, 15, 1265-1272.

Hünerbein Kv, Hamann HJ, Rüter E, Wiltschko W (2000). A GPS-based system for recording the flight paths of birds. Naturwissenschaften, 87, 278-279.

Icken W, Bennewitz J, Kalm E (2007). Analyse von Auktionsdaten der Pferdezucht und Einflussfaktoren auf die Preisbildung

Züchtungskunde, 79, 111 - 118.

Ipsos (2001). Marktanalyse der FN zum Pferdesport in Deutschland. http://www.wpsv.de/ipsos.htm. Abrufdatum: 17.03.2011.

Irvine C, Alexander S (1994). Factors affecting the circadian rythm in plasma concentrations in the horse. Domest Anim Endocrin, 11, 227-238.

Isenbügel E (2002). Vom Wildpferd zum Reitpferd. Schweizer Archiv für Tierheilkunde, 144, 7, 323-329.

Jørgensen GHM, Bøe KE (2007). A note on the effect of daily exercise and paddock size on the behavior of domestic horses (Equus caballus). Appl Anim Behav Sci, 107, 166-173.

Jussen U, Zeitler M, Groth W (1984). Untersuchungen über Haltungs- und Hygieneverhältnisse in bayrischen Pferdebeständen, 1. Mitteilung: Stallgebäude und Haltungssysteme. Züchtungskunde, 56, 199-208.

Kautzner J, Armonk NY (1995). Reproducibility of heart rate variability measurements. In: Malik M, Camm AJ (ed.): Heart rate variability. Futura Publ. Comp. Inc., S. 165-171.

Keeling $L$ (2009). An overview of the development of the welfare quality project assessment system. Welfare Quality Reports No. 12, http://www.welfarequality.net/everyone/43220/7/0/22. Abrufdatum: 15.03.2011.

Kiley-Worthington M (1987). The behaviour of horses in relation to management and training. J.A. Allen, London.

Kiley-Worthington M (1990). The behavior of horses in relation to management and training: towards ethologically sound environments. $J$ Equine Vet Sci (USA), 10, 62-71.

Kingston JK, Soppet GM, Rogers CW, Firth EC (2006). Use of a global positioning and heart rate monitoring system to assess training load in a group of Thoroughbred racehorses. Equine Vet J, 38, 106-109.

Klingel H (1972). Das Verhalten der Pferde (Equidae). Handb. Zool., 10, 168.

Korries OC (2003). Untersuchung pferdehaltender Betriebe in Niedersachsen. Bewertung unter dem Aspekt der Tiergerechtheit bei Trennung in verschiedene Nutzungsgruppen und Beachtung haltungsbedingter Schäden. Dissertation, Tierärztliche Hochschule Hannover.

Kownacki M, Sasimowski E, Budzynski M, Jezierski T, Kapron M, Jelen B (1978). Observations of 24-hours rhythm of natural behaviour of Polish primi- 
tive horses bred for conservation of genetic resources in a forest reverve. Genetica polonica, 19, 1, 61-77.

Krull HD (1984). Untersuchungen über die Aufnahme und Verdaulichkeit von Grünfutter beim Pferd. Dissertation, Tierärztliche Hochschule Hannover.

KTBL (2006). Nationaler Bewertungsrahmen Tierhaltungsverfahren. Methode zur Bewertung von Tierhaltungsanlagen hinsichtlich Umweltwirkungen und Tiergerechtheit. Herausgegeben vom Kuratorium für Technik und Bauwesen in der Landwirtschaft e.V. (KTBL), ISBN 978-3-939371-13-7.

Kumpula J, Colpaert A, Fielitz U (2001). GPS tracking and spatial data as a method for studying the use of pasture by reindeer. Conference "Tracking animals with GPS" Aberdeen 12-13 March 2001, 23.

Kusonose R, Hatakeyama H, Ichikawa F, Oki H, Asai Y, Ito K (1987). Behavioural studies on yearling horses in field environments 3 . Effects of the pasture shape on the behaviour of horses. B Equine Res Inst, 22, 1-5.

Kusunose R, Hatakeyama H, Ichikawa F, Oki H, Asai Y, Ito K (1987). Behavioural studies on yearling horses in field environments 3 . Effects of the pasture shape on the behaviour of horses. B Equine Res Inst, 22, 1-5.

Kuwahara M, Tsujino Y, Tsubone H, Kumagai E, Tsutsumi H, Tanigawa M (2004). Effects of pair housing on diurnal rhythms of heart rate and heart rate variability in miniature swine. Exp Anim (Tokyo), 53, 303-309.

Langbein J, Nürnberg G, Manteuffel G (2004). Visual discrimination learning in dwarf goats and associated changes in heart rate and heart rate variability. Physiol Behav, 82, 601-609.

Lavrenko EM, Karamysheva ZV (1993). Steppes of the Former Soviet Union and Mongolia. In: Coupland, R. T. (ed.). Ecosystems of the World. Natural Grasslands: Eastern Hemisphere and Resume. Elsevier, New York.

Lebelt D, Schönreiter S, Zanella AJ (1996). Salivary cortisol in stallions: the relationship with plasma levels, daytime profile and changes in response to semen collection. Pferdeheilkunde, 12, 411-414.

Lekeux P, Art T (1994). The respiratory system: anatomy, physiology, and adaptations to exercise and training. In: Hodgson, DR; Rose, RJ: The athletic horse: principles and practice of equine sports medicine. Saunders, ISBN: 9780721637594.

Levine MA (2005). Domestication and early history of the horse. In: Mills, DS; McDonnell, SM (2005): The domestic horse: the origins, development, and management of its behaviour. University Press, Cambridge.

Licht A (2000). Nicht-invasive Stressparameter beim Trabrennpferd. Dissertation, Ludwig-Maximilians-Universität München.

Liebetrau A (2004). Global Positioning System (GPS) - Herzfrequenzaufzeichnung, eine neue Hilfe für das Training von Fahrpferden. Dissertation, Tierärztliche Hochschule Hannover.

Lindner A, Offeney $F$ (1992). Einsatzdauer, Abgangsraten und Ursachen bei Sportpferden. Dtsch Tierärztl Wochenschr, 99, 39-42. 
Littlejohn A, Munro R (1972). Equine recumbency. Vet Rec, 90, 83-85.

Lugauer B (2010). Differences of movement pattern between Asiatic wild ass (Equus hemionus) and Przewalski's horse (Equus ferus przewalskii). Diplomarbeit, Universität Wien.

Mal ME, Friend TH, Lay DC, Vogelsang SG, Jenkins OC (1991). Behavioral responses of mares to short-term confinement and social isolation. Appl Anim Behav Sci, 31, 13-24.

Malpass JP, Weigler BJ (1994). A simple and effective environmental enrichment device for ponies in long-term indoor confinement. Contemporary Topics, 33, 74-76.

Marten J (1998). Artgerechte Pferdehaltung: Haltungsverfahren, Gebäude und Einrichtungen für Pferde. In: Fachgespräch Pferdehaltung, 25.11.1998, Arbeitsgemeinschaft Landtechnik und ländliches Bauwesen BadenWürttemberg e.V., 21-32.

Marten J (2000). Leitsatz: Bauliche Anlagen für die Pferdehaltung. KTBL Arbeitsblatt Nr. 1108 "Bauwesen und Tierhaltung. Bauen im ländlichen Raum. Spezielle Tierhaltung."

Martin-Rosset W, Vermorel M (1991). Maintenance energy requirement variations determined by indirect calorimetry and feeding trials in light horses. $\mathrm{J}$ Equine Vet Sci, 11, 42-45.

Mason GJ, Latham NR (2004). Can't stop, won't stop: is stereotypy a reliable animal welfare indicator? Anim Welfare, 13, 57-69.

Mayes E, Duncan P (1986). Temporal patterns of feeding in free-ranging horses. Behav, 96, 105-129.

McBride GE, Christopherson RJ, Sauer W (1985). Metabolic rate and plasma thyroid concentrations of mature horses in response to changes in ambient temperatures. Can J Anim Sci, 65, 375-382.

McDonnell SM (2002). Behaviour of horses. In: Jensen, P. (2002): The Ethology of Domestic Animals: An Introductory Text. CABI Publishing, Wallingford. ISBN: 0-85199-602-7.

McGreevy PD, Cripps PJ, French NP, Green LE, Nicol CJ (1995). Management factors associated with stereotypic and redirected behaviour in the thoroughbred horse. Equine Vet J, Vol. 27, 2, 82-83.

McGreevy PD, French NP, Nicol CJ (1995). The prevalence of abnormal behaviours in dressage, eventing and endurance horses in relation to stabling. Vet Rec, 137, 36-37.

Mehl H (1996). GPS - Global Positioning System. Informatik Spektrum, 19, 33-34.

Meyer H, Coenen M (2002). Pferdefütterung. 4. Auflage, Blackwell Wissenschafts-Verlag, Berlin, Wien.

Mohr E, Langbein J, Nürnberg G (2002). Heart rate variability: A noninvasive approach to measure stress in calves and cows. Physiol Behav, 75, 251-259. 
Mooney DM, Groome LJ, Bentz LS, Holland S (1995). Poincaré analysis of fetal heart rate pattern: effect of observation period. Engineering in medicine and biology society IEEE 17th annual conference, Montreal, Que., Canada.

Morgan K (1998). Thermoneutral zone and critical temperature of horses. J Thermal Biol, 23, 59-61.

Morgan K, Ehrlemark A, Sallvik K (1997). Dissipation of heat from standing horses exposed to ambient temperatures between $-3^{\circ} \mathrm{C}$ and $37^{\circ} \mathrm{C}$. J Thermal Biol, 22, 177-186.

Möstl E, Palme R (2002). Hormones as indicators of stress. Domest Anim Endokrin, 23, 67-74.

Mück-Weymann M (2002). Die Herzratenvariabilität als globaler Adaptivitätsfaktor in psycho-neuro-kardialen Funktionskreisen. In: Mattke D (Hrsg.) Vom Allgemeinen zum Besonderen: Störungsspezifische Konzepte und Behandlung in der Psychosomatik. Verlag für Akademische Schriften, Frankfurt/Main, 322-327.

Müller R, Schrader $L$ (2003). A new method to measure behavioural activity levels in dairy cows. Appl Anim Behav Sci, 83, 247-258.

Naguib M (2006). Methoden der Verhaltensbiologie. Springer Verlag, Berlin Heidelberg. ISBN: 978-3-540-33494-7.

Nagy K, Bodó G, Bárdos G, Harnos A, Kabai P (2009). The effect of a feeding stress-test on the behaviour and heart rate variability of control and cribbiting horses (with or without inhibition). Appl Anim Behav Sci, 121, 140-147.

Norman SE, Eager RA, Waran NK, Jeffery L, Schroter RC, Marlin DJ (2005). Recording of ECG signals on portable MiniDisc recorder for time and frequency domain heart rate variability analysis. Physiol Behav, 83, 729-738.

Osterhues HH, Hanzel SR, Kochs M, Hombach V (1997). Influence of physical activity on 24-h measurements of heart rate variability in patients with coronary artery disease. Am J Cardiol, 80, 1434-1437.

Pedersen GR, Sondergaard E, Ladewig J (2004). The influence of bedding on the time horses spend recumbent. J Equine Vet Sci, 24, 4, 153-158.

Petersen S, Tölle KH, Blobel K, Grabner A, Krieter J (2006). Erhebungen zur Pferdehaltung in Pensionsbetrieben Schleswig-Holsteins. Züchtungskunde, 78, 207 - 217.

Pick M (1986). Handbuch der Pferdekrankheiten. Frankh'sche Verlagsbuchhandlung, Stuttgart. ISBN: 3440059588.

Ralston SL, Freeman DE, Baile CA (1983). Volatile fatty acids and the role of the large intestine in the controle of feed intake in ponies. J Anim Sci, 57, 815-825.

Riegel RJ, Hakola SE (2002). Bild-Text-Atlas zur Anatomie und Klinik des Pferdes. Schlütersche GmbH \& Co. KG, Hannover. ISBN: 3-87706-652-6.

Rietmann TR, Stauffacher M, Bernasconi P, Auer JA, Weishaupt MA (2004). The association between heart rate, heart rate variability, endocrine and be- 
havioural pain measures in horses suffering from laminitis. $J$ Vet Med $A$ Physiol Pathol Clin Med, 51, 218-225.

Rodewald A (1989). Fehler bei der Haltung und Nutzung als Schadensursache bei Pferden in Reitbetrieben. Dissertation, Universität München.

Ruckebusch Y (1975). The hypnogram as an index of adaptation of farm animals to changes in their environment. Appl Anim Ethol, 2, 3-18.

Safran MR, Garrett WE, Seaber AV, Glisson RR, Ribbeck BM (1988). The role of warmup in muscular injury prevention. Am J Sports Med, 16, 123-129.

Schäfer M (1978). Pferd. In: Sambraus, H.H. (1978): Nutztierethologie. Das Verhalten landwirtschaftlicher Nutztiere - eine angewandte Verhaltenskunde für die Praxis. Paul Parey Verlag, Berlin, Hamburg.

Schäfer M (1991). Ansprüche des Pferdes an seine Umwelt. In: Pferdehaltung. Hrsg.: Pirkelmann, H. (1991), Ulmer Verlag, Stuttgart.

Schlichting KE (2001). Atemwegserkrankungen. Reitsportmagazin, 3, 22-23.

Shellock FG, Prentice WE (1985). Warming-up and stretching for improved physical performance and prevention of sports-related injuries. Sports Med, 2, 267-78.

Singer DH, Ori Z (1995). Changes in heart rate variability associated with sudden cardiac death. In: Malik M, Camm AJ (ed.): Heart rate variability. Futura Publ. Comp., Inc., S. 429-448.

Stefanski V, Hendrichs H (1996). Social confrontation in male guinea pigs: Behavior, experience, and complement activity. Physiol Behav, 60, 235-241.

Steiner I, Bürgi C, Werffeli S, Dell'Omo G, Paolo Valenti P, Tröster G et al. (2000). A GPS logger and software for analysis of homing in pigeons and small mammals. Physiol Behav, 71, 589-596.

Sweeting MP, Houpt CE, Houpt KA (1986). Social facilitation of feeding and time budgets in stabled ponies. J. Anim. Sci., 60, 369-374.

Task Force (1996). Task Force of the European Society of Cardiology and North American Society of Pacing and Electrophysiology. Heart rate variability: standards of measurement, physiological interpretation, and clinical use. Eur Heart J, 17, 354-381.

Tetzner K (2008). Mehrjähriges Konditionstraining von Sportpferden : Eine longitudinale Auswertung von Kraft-, Ausdauer-, und Schnelligkeitstraining anhand von Laktat, Herzfrequenz und Geschwindigkeitsbeziehungen. Dissertation, Tierärztliche Hochschule Hannover.

Tigges F (2009). Eine empirische Studie zur Bewertung von Pferdehaltungssystemen durch eine Befragung von Züchtern und Reitern. Tagungsband Göttinger Pferdetage 2009, FN-Verlag, Warendorf. ISBN: 987-3-88542-7421.

Tottewitz F (2005). Untersuchungen zum Raum- und Zeitverhalten von Rotwild durch GPS-GSM Telemetrien der Schorfheide. BFH-Nachrichten 4/2005,

Tottewitz F, Neumann M, Sparing H (2010). Lebensraumnutzung von Rotwild in der Schorfheide - Ergebnisse aus mehrjährigen GPS-GSM- 
Satellitentelemetriestudien. In: Aktuelle Beiträge zur Wildökologie und Jagdwirtschaft in Brandenburg. Eberswalder Forstliche Schriftenreihe Band 45, 94-106.

Turner LW, Udal MC, Larson BT, Shearer SA (2000). Monitoring cattle behaviour and pasture use with GPS and GIS. Can J Anim Sci, 80, 405-413.

Tyler SJ (1972). The behaviour and social organization of the New Forest Ponies. Anim Beh Monogr 5, 87-193.

Vandenput S, Votion D, Duvivier DH, Van Erck E, Anciaux N, Art T et al. (1998). Effect of set stabled environmental control on pulmonary function and airway reactivity of COPD affected horses. Vet J, 155, 189-195.

Vecchiotti GG, Galanti R (1987). Evidence of heredity of cribbing, weaving and stall walking. Livestock Prod Sci, 14, 91-95.

Visser EK, Van Reenen CG, Van der Werf JTN, Schilder MBH, Knaap JH, Barneveld $A$ et al. (2002). Heart rate and heart rate variability during a novel object test and handling test in young horses. Physiol Behav, 76, 289-296.

von Borell E, Langbein J, Després G, Hansen S, Leterrier C, Marchant-Forde $J$ et al. (2007). Heart rate variability as a measure of automatic regulation of cardiac activity for assessing stress and welfare in farm animals - A review. Physiol Behav, 92, 293-316.

Voss B, Mohr E, Krzywanek H (2002). Effects of aqua-treadmill exercise on selected blood parameters and on heart-rate variability of horses. J Vet Med A, 49, 137-143.

Wallin L, Strandberg E, Phillipson J, Dalin G (2000). Estimates of longevity and causes of culling and death in Swedish warmbloods and coldblood horses. Livest Prod Sci, 63, 275-289.

Wark T, Corke P, Sikka P, Klingbeil L, Guo Y, Crossman C et al. (2007). Transforming agriculture through pervasive wireless sensor networks. Pervasive Computing, IEEE Computer Society, 50-57.

Werhahn H, Hessel EF, Van den Weghe HFA (in press). Auswirkungen unterschiedlicher Auslaufgestaltungen auf die Bewegungsaktivität während des Auslaufes von Sportpferden in Einzelhaltung. In: 10. Tagung Bau, Technik und Umwelt in der landwirtschaftlichen Nutztierhaltung 2011. 27.-30.09.2011, Kiel, angenommen 17/03/2011.

Widmann U (1990). Fortbewegung von Pferden außerhalb der Nutzungszeit. Diplomarbeit, Universität Hohenheim.

Winskill LC, Waran NK, Young RJ (1996). The effect of a foraging device (a modified "Edinburgh Foodball") on the behaviour of the stabled horse. Appl Anim Behav Sci, 48, 425-429.

Zeeb K (1981). Basic behavioural needs of horses. Appl. Anim. Ethol., 7, 391-392.

Zeitler-Feicht MH Auflage (2008). Handbuch Pferdeverhalten. Ursache, Therapie und Prophylaxe von Problemverhalten. Verlag Eugen Ulmer GmbH \& Co., Stuttgart (Hohenheim). ISBN 978-3800155798. 
Zogg JM (2009). GPS und GNSS: Grundlagen der Ortung und Navigation mit Satelliten. Ublox Users Guide, Ublox AG, Thalwil CH, 43-61. 


\section{Anhang}

\subsection{Bewertungsbögen}

\subsubsection{Verhalten während des Auslaufs}

\section{Datum:}

\section{Einzelauslauf:}

2er-Gruppe:

Äußere Umstände/Besonderheiten:

Scan sampling (alle 5 Min.)

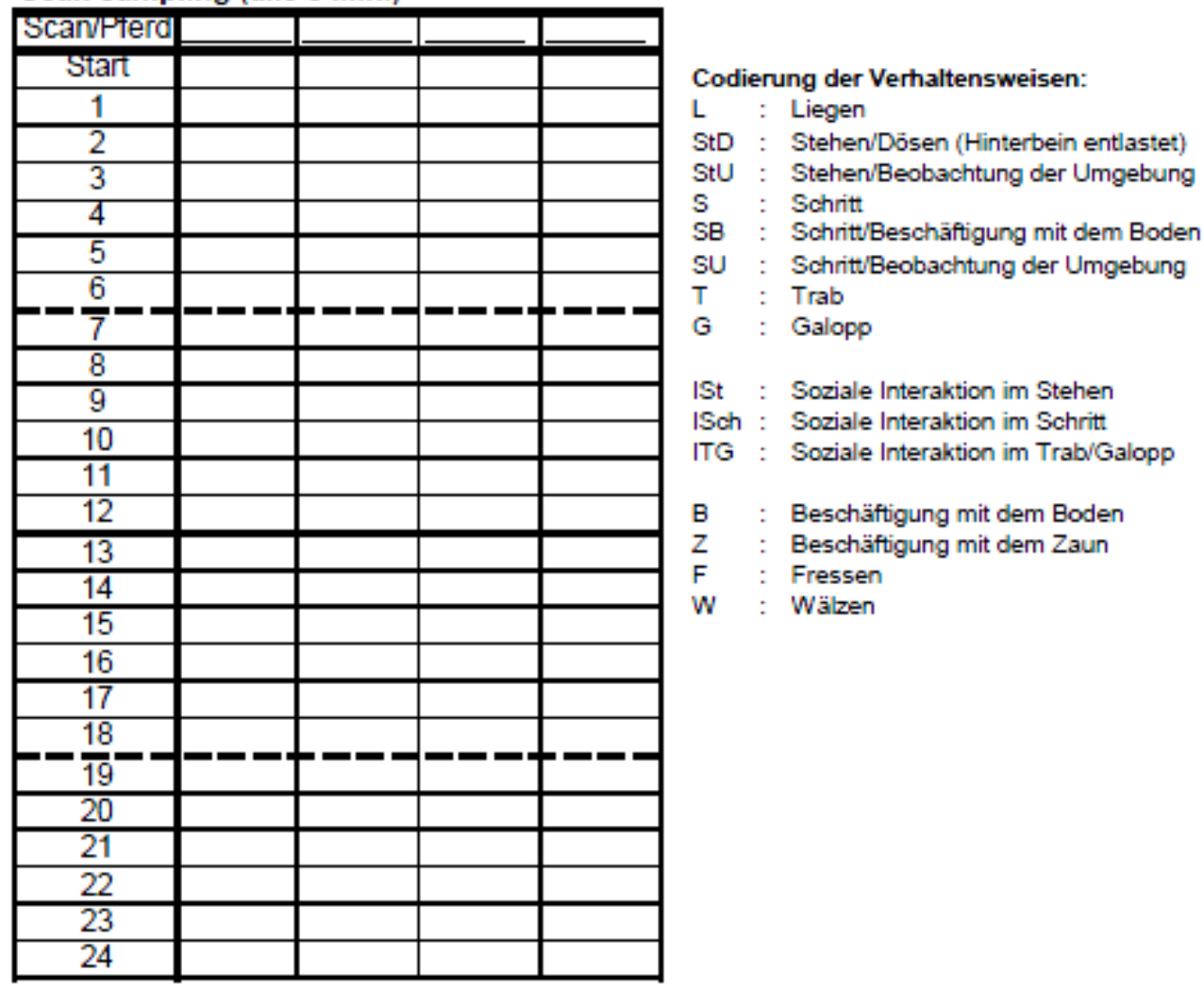

Gesamteindruck des Verhaltens (Skala: 1 bis 5 )

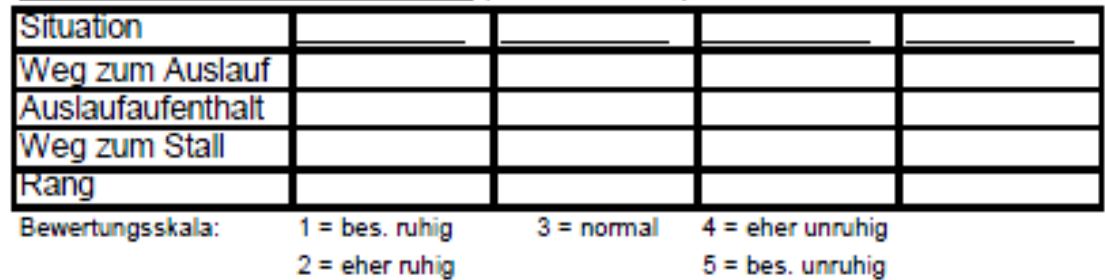




\subsubsection{Verhalten während des Trainings}

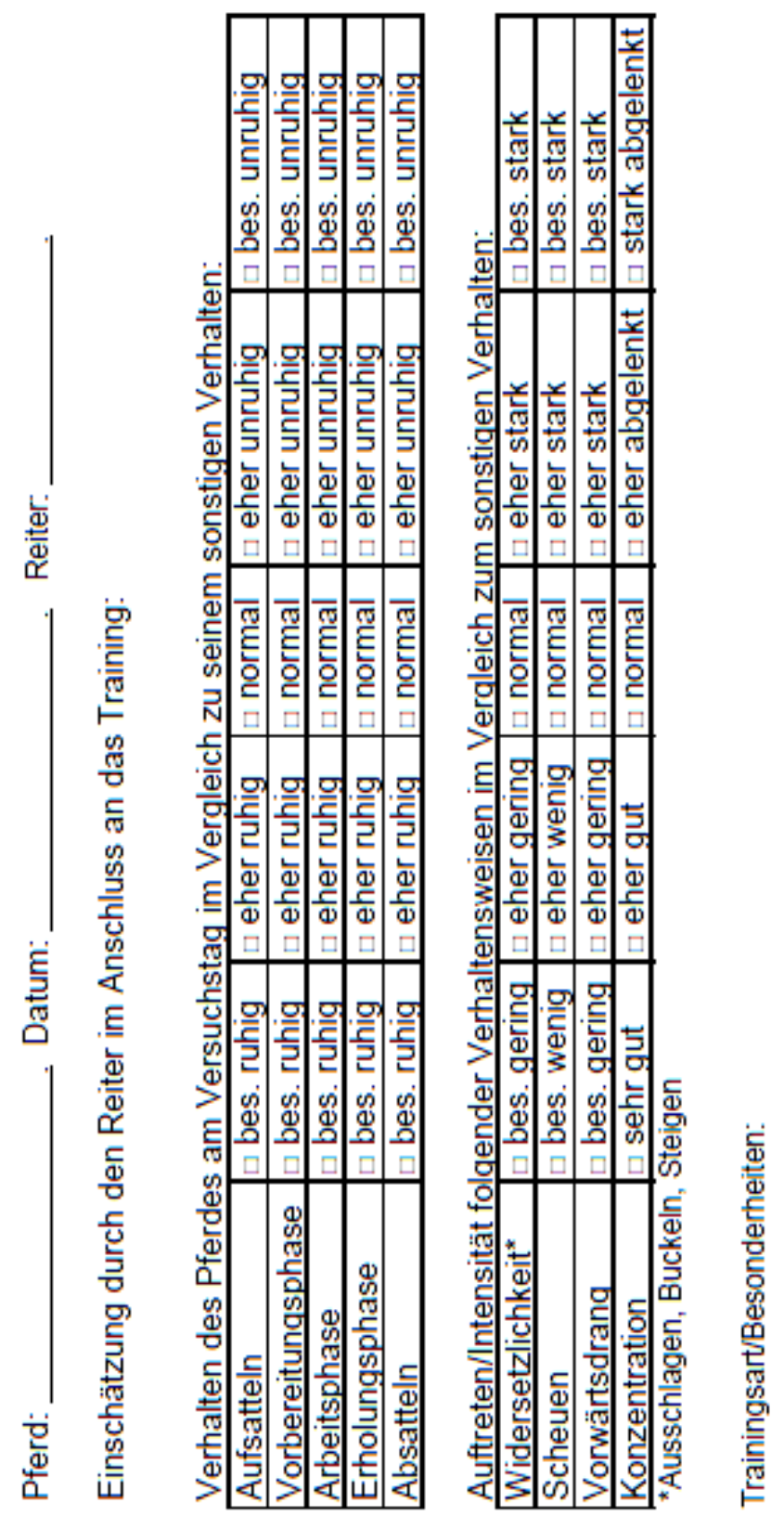


8.2. Lebenslauf (Stand: 03.08.2011)

\begin{tabular}{|c|c|}
\hline Anschrift: & $\begin{array}{l}\text { Hanna Werhahn } \\
\text { Oldendorfer Weg } 6 \\
29553 \text { Bienenbüttel }\end{array}$ \\
\hline Telefon: & 05823-313 \\
\hline Mobil-Tel.: & 0176-20132585 \\
\hline E-Mail: & hanna.we@web.de \\
\hline Geburtstag: & 02. Mai 1984 \\
\hline Geburtsort: & Uelzen-Veerßen \\
\hline
\end{tabular}

\section{Ausbildung}

11/2008 - 07/2011 Promotion an der Georg-August-Universität Göttingen zu dem Thema „Bewegungsbedarf von Sportpferden in Einzelhaltung" in der Abteilung Verfahrenstechnik in der Veredelungswirtschaft

10/2006-10/2008 Masterstudium der Agrarwissenschaften mit dem Schwerpunkt Nutztierwissenschaften an der Georg-AugustUniversität Göttingen

10/2003-07/2006 Bachelorstudium der Agrarwissenschaften mit dem Schwerpunkt Nutztierwissenschaften an der Georg-AugustUniversität Göttingen

\begin{tabular}{|c|c|}
\hline beitserfahrung & \\
\hline ab $7 / 2011$ & $\begin{array}{l}\text { Büroaushilfe bei der Firma Redell Arbeitssicherheit, } \\
\text { Lüneburg }\end{array}$ \\
\hline $11 / 2008-07 / 2011$ & $\begin{array}{l}\text { Wissenschaftliche Mitarbeiterin an der Georg-August- } \\
\text { Universität Göttingen, Abteilung Verfahrenstechnik in der } \\
\text { Veredelungswirtschaft }\end{array}$ \\
\hline 07/2003 - 08/2008 & $\begin{array}{l}\text { In diesem Zeitraum 4-mal für jeweils } 3 \text { bis } 8 \text { Wochen: } \\
\text { Working Student im Turnierstall Bettina und Andrew Hoy, } \\
\text { Gatcombe Park, Gloucestershire, England }\end{array}$ \\
\hline 06/2008 - 07/2008 & $\begin{array}{l}\text { Studentische Hilfskraft an der Georg-August-Universität } \\
\text { Göttingen, Abteilung Verfahrenstechnik in der } \\
\text { Veredelungswirtschaft }\end{array}$ \\
\hline $11 / 2007$ u. 03/2006 & Messe-Hostess (Agritechnica, CeBIT) \\
\hline seit 2000 & regelmäßiges Erteilen von Reitunterricht und Beritt \\
\hline
\end{tabular}




\section{Praktika}

08/2007 - 09/2007 Praktikum bei der Firma Sano - Moderne Tierernährung, Grafenwald

03/2007-03/2007 Praktikum bei Michael Beihsner (praktizierender Tierarzt), Ebergötzen

02/2005 - 04/2005 Praktikum bei der Landwirtschaftskammer Niedersachsen, Bezirksstelle Uelzen

08/2004-10/2004 Praktikum auf dem Milchvieh- und Ackerbaubetrieb Ernst August Henneke, Elbickerode

03/2000 - 03/2000 Schulpraktikum bei der Firma Bioplant, Ebstorf

\section{Schule}

08/1996 - 06/2003 Lessing-Gymnasium Uelzen

08/1994 - 07/1996 Orientierungsstufe Bad Bevensen

08/1990 - 07/1994 Grundschule Bienenbüttel

\section{Zusätzliche Qualifikationen}

Sprachen: Deutsch (Muttersprache), Englisch (verhandlungssicher), Französisch (Grundkenntnisse)

EDV-Kenntnisse: MS Office Anwendungen, SAS

Berufs- und arbeitspädagogische Eignung („BAP-Schein“)

Fahrerlaubnisklassen $\mathrm{B}, \mathrm{BE}, \mathrm{T}$

Trainer-C-Lizenz Reiten

Hobbys: Reitsport (Erfolge in der Dressur bis Klasse S), Lesen, Sport allgemein 


\subsection{Veröffentlichungen}

\subsubsection{Wissenschaftliche Beiträge mit Begutachtung}

WERHAHN, H.; HESSEL, E.F.; BACHHAUSEN, I.; VAN DEN WEGHE, H.F.A. (2010): Effects of different bedding materials on the behavior of horses housed in single stalls. Journal of Equine Veterinary Science Vol. 30, No. 8, 425-431. [siehe S. XXV-XXXI]

WERHAHN, H.; HESSEL, E.F.; SCHULZE, H.; VAN DEN WEGHE, H.F.A. (2011): Temporary turnout for free exercise in groups: effects on the behaviour of competition horses housed in single stalls. Journal of Equine Veterinary Science Vol. 31, 417-425. [siehe S. 25-33]

WERHAHN, H.; HESSEL, E.F.; VAN DEN WEGHE, H.F.A. (in press): Competition horses housed in single stalls (1st communication): Behaviour and activity patterns during free exercise according to its configuration. Journal of Equine Veterinary Science (accepted 13/06/2011). [siehe S. 3554]

WERHAHN, H.; HESSEL, E.F.; VAN DEN WEGHE, H.F.A. (in press): Competition horses housed in single stalls ( $2^{\text {nd }}$ communication): Effects of free exercise on the behaviour in the stable and during training and on the degree of stress. Journal of Equine Veterinary Science (accepted 21/04/2011). [siehe S. 56-81] 


\subsubsection{Tagungsbeiträge}

WERHAHN, H.; HESSEL, E.F.; BACHHAUSEN, I.; VAN DEN WEGHE, H.F.A. (2009): Einfluss verschiedener Einstreumaterialien auf das Verhalten von Pferden in Boxenhaltung. In: 9. Tagung Bau, Technik und Umwelt in der landwirtschaftlichen Nutztierhaltung 2009. 21.-23.09.2009, Berlin, 184-189. ISBN 978-3-941583-27-6.

WERHAHN, H.; HESSEL, E.F.; SCHULZE, H.; VAN DEN WEGHE, H.F.A. (2010): Zeitlich begrenzte freie Bewegungsmöglichkeit in Gruppen: Auswirkungen auf das Verhalten von Sportpferden in Einzelhaltung. In: 15. Internationale Fachtagung zum Thema Tierschutz, 24.-27. Februar 2010 in Nürtingen. In: Tagung der DVG-Fachgruppen Tierschutz und Versuchstierkunde. Verlag DVG Service GmbH, Gießen, S. 257-261. ISBN 978-3-941703-59-9.

WERHAHN, H.; HESSEL, E.F.; VAN DEN WEGHE, H.F.A. (2011): Sportpferde in Einzelhaltung: Auswirkungen des Auslaufmanagements auf das Verhalten und die Stressbelastung. In: Göttinger Pferdetage '11: Zucht, Haltung und Ernährung von Sportpferden. 31.3.-1.4.2011 in Göttingen. FNVerlag, Warendorf, S. 122-123. ISBN: 978-3-88542-758-2.

WERHAHN, H.; HESSEL, E.F.; SCHULZE, H.; VAN DEN WEGHE, H.F.A. (2011): Effect of free exercise in groups on the behaviour of competition horses housed in single stalls. In: XVth International Congress on Animal Hygiene 2011: Animal Hygiene and Sustainable Livestock Production, July 37, 2011, Vienna, Austria. Tribun EU s.r.o., Brno. ISBN: 978-80-263-0008-3.

WERHAHN, H.; HESSEL, E.F.; VAN DEN WEGHE, H.F.A. (in press): Effekte der Auslaufgestaltung auf die Herzfrequenzvariabilität (HRV) und das Verhalten von Sportpferden in Einzelboxen. In: 10. Tagung Bau, Technik und Umwelt in der landwirtschaftlichen Nutztierhaltung 2011. 27.-30.09.2011, Kiel. Angenommen 17/03/2011.

WERHAHN, H.; HESSEL, E.F.; VAN DEN WEGHE, H.F.A. (in press): Auswirkungen unterschiedlicher Auslaufgestaltungen auf die Bewegungsaktivität während des Auslaufes von Sportpferden in Einzelhaltung. In: 10. Tagung Bau, Technik und Umwelt in der 
landwirtschaftlichen Nutztierhaltung 2011. 27.-30.09.2011, Kiel. Angenommen 17/03/2011.

\subsubsection{Sonstige Veröffentlichungen}

WERHAHN, H.; HESSEL, E.F.; VAN DEN WEGHE, H.F.A. (2009): Einfluss verschiedener Einstreumaterialien auf das Verhalten von Pferden in Boxenhaltung. Landtechnik 64, 238-241. 


\section{DANKSAGUNG}

Mein herzlicher Dank gilt allen, die auf so vielfältige Weise zum Gelingen dieser Arbeit beigetragen haben.

Herrn Prof. Dr. Ir. H. Van den Weghe für das Vertrauen, mir diese Arbeit zu überlassen und die finanzielle Unterstützung.

Frau Prof. Dr. E. Hessel für die Betreuung der Arbeit, ständige Diskussionsbereitschaft, fachliche Unterstützung bei der statistischen Auswertung und Veröffentlichung der Ergebnisse und angeregte Gespräche auf den zahlreichen Fahrten nach Vechta.

Herrn Prof. Dr. Dr. M. Gauly für die Übernahme des Korreferates und seine wertvolle Kritik „von außen“.

Den Mitarbeitern der Außenstelle Vechta, die mich trotz räumlicher Entfernung so herzlich aufgenommen und tatkräftig unterstützt haben. Ganz besonders möchte ich mich bei Hartmut bedanken für die technische Unterstützung der Versuche, die immer wieder die Lösung unlösbarer Probleme erforderte und auch vor seinem Urlaub nicht immer Halt machte. Außerdem bei Felix für ein immer offenes Ohr, die unkomplizierte Zusammenarbeit, intensive (Fach-)Gespräche und ständige Hilfsbereitschaft und Motivation.

Den Mitarbeitern der Abteilung Agrartechnik, die mich als „Pferdetussi“ nicht nur geduldet, sondern herzlich aufgenommen und immer über die „Departments- und Abteilungsgrenzen“ hinweg unterstützt haben. Ein besonderer Dank geht an Ulli für die harmonische, lustige, philosophische und motivierende Bürogemeinschaft und sein ständig offenes Ohr in allen Lebenslagen.

Den Leitern der Praxisbetriebe Ludwig Hecke und Niels von Hirschheydt für die Offenheit gegenüber den Versuchen. Ebenso ihren Teams für die gute Zusammenarbeit und den Besitzern der Pferde für das entgegen gebrachte Vertrauen.

„Meinen“ Studentinnen Hanna, Meike und Nicole für die zuverlässige Unterstützung bei der Durchführung der Versuche.

Sabine für das Korrekturlesen der Arbeit.

Zu guter Letzt geht noch ein ganz besonderer Dank an meine Eltern und an Onno. Onno danke ich für seinen stets kritischen und aufmunternden Rückhalt in allen „Doktorarbeitslaunen“. Meinen Eltern möchte ich von ganzem Herzen für ihre uneingeschränkte Unterstützung während meiner gesamten Ausbildung danken. Euch ist diese Arbeit gewidmet. 